\title{
Oil utilization degree at various pore sizes via different displacement methods
}

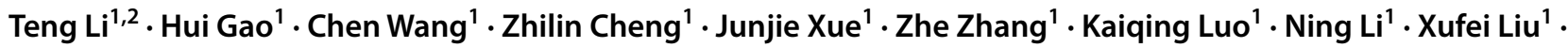 \\ Jiangfeng $\mathrm{Cao}^{1}$
}

Received: 29 November 2021 / Accepted: 18 January 2022 / Published online: 31 January 2022

(c) The Author(s) 2022

\begin{abstract}
A reasonable displacement method is essential to improve the oil displacement efficiency of tight reservoirs. In this study, three different displacement methods were utilized on the tight core samples obtained from the Yanchang Formation Chang 8 and Chang 9 tight oil reservoirs: spontaneous imbibition displacement, various water flooding rate displacement and water flooding displacement after spontaneous imbibitions; furthermore, the oil utilization degree of the residual oil in various pores was discussed. The oil displacement efficiency of the spontaneous imbibitions was approximately $26.91 \%$ and $29.56 \%$ for the Chang 8 and Chang 9 samples, respectively. With an increasing water flooding rate, the oil displacement efficiency features an inverse "V"-like tendency, and a water flooding rate of $0.06 \mathrm{~mL} / \mathrm{min}$ was the optimal value as; the oil displacement efficiency achieved was $63.56 \%$ and $60.27 \%$ for the Chang 8 and Chang 9, respectively. When compared with spontaneous imbibitions, at a displacement rate of $0.06 \mathrm{~mL} / \mathrm{min}$ after spontaneous imbibition, the oil displacement efficiency could be further increased to $50.02 \%$ and $30.35 \%$, respectively. The differences in the oil displacement efficiency using various displacement methods are primarily related to the degree of utilization of residual oil in various pores. The progressively refined pore classification method is used to study the degree of oil utilization in various pores, and the pores in the tight reservoir can be divided into four types: P1, P2, P3 and P4. Regarding the spontaneous imbibition displacement, the displacement of the residual oil is dominantly determined by the residual oil present in the P2 and P3 pores; as the residual oil massively accumulates in the $\mathrm{P} 2$ pores, the discharging of the residual oil in this part finally determines the oil displacement efficiency when the water flooding rates changed. At a water flooding rate of $0.06 \mathrm{~mL} / \mathrm{min}$ after spontaneous imbibition, the oil utilization degree of residual oil in various pores is enhanced, particularly for the P2, P3, and P4 pores, and the pore structure contributes to the increase in the oil displacement efficiency. Using the progressively refined pore classification method, the complexity of the distribution of residual oil in tight reservoirs could be studied quantitatively and elaborately, and the results can efficiently guide the development of residual oil in tight oil reservoirs.
\end{abstract}

Keywords Tight oil · Progressively refined pore classification method · Pore types · Oil utilization degree

\section{Introduction}

Low- and ultra-low-permeability tight oil reserves are abundant in China. These are attractive but difficult to develop. Nanoscale pores are developed in tight sandstone reservoir,

Hui Gao

ghtopsun1@163.com

1 College of Petroleum Engineering, Xi' an Shiyou University, Xi' an 710065, Shaanxi, China

2 Key Laboratory of Coalbed Methane Resources \& Reservoir Formation Process, Ministry of Education, China University of Mining and Technology, Xuzhou 221116, Jiangsu, China leading to a poor reservoir quality, complex pore structure, and strong reservoir heterogeneity. A refined study on the distribution, occurrence states, occurrence quantity, and degree of tight oil use is essential to enhance tight oil production.

Conventional water flooding displacement is limited to tight sandstone reservoirs, and spontaneous imbibition is key for the enhancement of tight oil recovery. Studies on the spontaneous imbibition displacement of tight oil are currently abundant, and they primarily focus on theoretical derivation (Handy 1960; Cryer 1963; Krohn 1988), measurement methods (Li and Horne 2001; Ge et al. 2015; Gao and $\mathrm{Hu}$ 2016), and numerical simulation (Hammecker

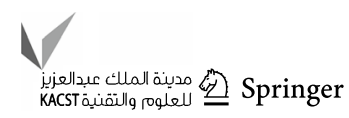


et al. 1993; Kresse and Hafner 1993; Constantinides and Payatakes 1996; Martic et al. 2002; Hatiboglu and Babadagli 2008; Palma et al. 2015; Khajepor et al. 2019), among others. Spontaneous imbibition includes both static and dynamic imbibition. For the former, the capillary force is the dominant driving force (Zeng et al. 2020), a positive relationship exists between the water adsorption volume and the square root of time (Handy 1960), and the water adsorption time is related to the pore structure (Mattax and Kyte 1962). Dynamic imbibition occurrences in the microfractures in tight sandstone reservoirs (Shen et al. 2016; Yang et al. 2016; Dai et al. 2019), molecular dynamics simulation (Kresse and Hafner 1993; Martic et al. 2002), Boltzmann simulation (Kresse and Hafner 1993; Hatiboglu and Babadagli 2008; Palma et al. 2015; Khajepor et al. 2019), and pore network simulation (Constantinides and Payatakes 1996) are effective methods for studying dynamic imbibition. During the production of tight oil in oilfields, the use of a reasonable water flooding displacement rate after spontaneous imbibition is an effective method for improving the oil displacement efficiency (Wang et al. 2018).

The characteristics of the pore structure are essential parameters that determine the distribution of residual oil and the mobility of fluid in a tight reservoir. Currently, various methods can be used to describe the pore structure of tight reservoirs, and low-field nuclear magnetic resonance (NMR) is commonly used in unconventional oil and gas fields. NMR has an individual advantage for describing the micro-pore structure (such as the properties of the reservoir, pore throat characteristics, and pore size distribution), and the fluid in the pore (such as the volume of movable fluid, saturation of movable oil, and distribution of residual oil) (Hamada and AbuShanab 2008; Yang et al. 2013; Lai et al. 2018; Lyu et al. 2018a; Gao et al. 2019; $\mathrm{Li}$ et al. 2019; Wu et al. 2022). The pores in a tight reservoir can be classified according to the transverse relaxation time $\left(T_{2}\right)$, Peng et al. (2018) reported that the pores could be divided into micro-pore $\left(T_{2} \leq 30 \mathrm{~ms}\right)$, mesopore (30-90 ms), macropore (90-200 ms) and super macropore $\left(T_{2}>200 \mathrm{~ms}\right)$. According to the fluid movement in the pores, the pores can be classified into completely unmovable pores, partially movable pores, and completely movable pores (Wang et al. 2021). Four characteristic points in the NMR $T_{2}$ spectrum were selected, and the pores were divided into nanopores, micro-pores, meso-pores and macropores (Dai et al. 2019). Using mercury intrusion porosimetry (MIP) and NMR, $T_{2}$ can be transferred to the pore aperture, and the pore structure can be comprehensively described (Volokitin et al. 2001; Lyu et al. 2018b; Dai et al. 2019; Chen et al. 2020). The short components of $T_{2}$ generally respond to pores with smaller sizes, and the long components of $T_{2}$ respond to large pores in tight reservoirs (Wu et al. 2018). Using this method, the pores can be divided into microscale macropores $(>10 \mu \mathrm{m})$, microscale micro-pore $(1-10 \mu \mathrm{m})$, submicro-pores $(0.1-1 \mu \mathrm{m})$ and nanopores $(<0.1 \mu \mathrm{m})$ (Hu et al. 2020). The submicropores contribute to oil displacement efficiency, whereas the nanopores contribute to oil displacement via spontaneous imbibitions (Cheng et al. 2019). During the oil displacement procedures, oil occurrences in the microscale macropores contribute dominantly to the enhancement of oil recovery (Chen et al. 2020).

The pores in a tight reservoir exhibit self-similarity. Compared with classical European geometry, the fractal dimension can describe the heterogeneity and complexity of pores (Krohn and Thompson 1986; Krohn 1988; Jin et al. 2017a, b; Guo et al. 2019; Wang et al. 2019a, b, 2020, 2021; Wu et al. 2020, 2022; Yang et al. 2021). Pores with fractal dimensions below 2 and above 3 have a weak influence on the pore structure (Guo et al. 2019). Tight reservoirs can be divided into four types, according to the fractal dimension: Type I contains large amounts of dissolved-intergranular pores and original intergranular pores; type II features dissolved-intergranular pores; and the type III and IV contain dissoloveintragranular pores and micro-pores in clay matrix, respectively (Guo et al. 2020). The fractal dimension reflects the quality of the tight reservoirs. The smaller the pore aperture, the larger the fractal dimension, the more complex the pore structure, and the lower the permeability (Wang et al. 2019a, 2020). Wang et al. (2019b) reported that when the fractal dimension exceeded 2.586, a tight reservoir did not favor oil accumulation. If the fractal dimension was approximately 3 , the porosity and permeability of the tight reservoir would decrease significantly (Wang et al. 2021). The fractal dimensions of submicro-pores and nanopores commonly reflect the complexity of the pores, and the fractal dimensions of the microscale macropores feature significantly influence the permeability of tight reservoirs (Hu et al. 2020). The fractal dimension of partially movable pores and completely movable pores contributes to enhancement of the oil displacement (Wu et al. 2020; Wang et al. 2021).

There have been numerous researches on the characteristics of the pore structures and movable fluid using NMR and fractal methods. However, the distribution characteristics of various types of pores using various displacement methods are unclear. In this study, Yanchang Formation tight sandstone samples were collected from the Ordos Basin, and oil displacement measurements using three different displacement methods (spontaneous imbibition, water flooding with various rates and water flooding after the spontaneous imbibitions) were carried out to investigate the oil displacement characteristics. Combined with the fractal theory, a new pore classification method of the residual oil, which is named as the progressively refined pore classification method, is provided. Furthermore, the oil utilization degree of tight oil in various pores was comprehensively studied. 


\section{Samples and method}

\section{Samples}

Tight sandstone samples were collected from the Yanchang Formation Chang 8 member and Chang 9 members, northeast of the Ordos Basin, China. The Chang 8 and Chang 9 members are tight reservoirs. The porosity of Chang 8 member ranged from 6.71 to $14.56 \%$ (with an average of $9.81 \%$ ), and its permeability varied from $0.05 \times 10^{-3} \mu \mathrm{m}^{2}$ to $2.99 \times 10^{-3} \mu \mathrm{m}^{2}$ (with an average of $0.74 \times 10^{-3} \mu \mathrm{m}^{2}$ ). As for the Chang 9 member, the porosity and permeability ranged from 12.3 to $15.18 \%$ (with an average of $13.81 \%$ ) and $0.03 \times 10^{-3} \mu \mathrm{m}^{2}$ to $2.38 \times 10^{-3} \mu \mathrm{m}^{2}\left(0.53 \times 10^{-3} \mu \mathrm{m}^{2}\right)$, respectively. The tight reservoirs featured strong heterogeneity, and the tight core samples were collected from a nearby region in the full-diameter cores at the horizontal stratification direction. The core samples were polished to ensure that the planeness of the end face was less than $0.01^{\circ}$ prior to the measurements. The basic reservoir parameters are presented in Table 1.

\section{Measurement procedures}

Spontaneous imbibition measurements were carried out using a BLHH-6 N digital display of an electric thermal constant-temperature water bath, and the maximum temperature measured reached $100{ }^{\circ} \mathrm{C}$ with an accuracy of $0.5^{\circ} \mathrm{C}$. Oil displacement measurements were conducted using an LDY150 displacement seepage instrument, which included a flat flow pump, a thermostat, a core holding unit, and an intermediate container. The flow rate ranged from $0.01 \mathrm{~mL} / \mathrm{min}$ to $10 \mathrm{~mL} / \mathrm{min}$ and was controlled by the flat flow pump; the temperature of the thermostat was maintained at $100{ }^{\circ} \mathrm{C}$, and the temperature sensitivity could be controlled at $0.1{ }^{\circ} \mathrm{C}$; the maximum pressure in the core holding unit and intermediate container could reach 50 and $30 \mathrm{Mpa}$, respectively. Three intermediate containers, which contained simulated oil, an $\mathrm{MnCl}_{2}$ solution, and a simulated stratum water, were used in this study. The NMR measurements before and after the displacement measurements were carried out using a Geospec2/53 NMR spectrometer at a frequency of $4.40 \mathrm{MHz}$.

Table 1 Basic reservoir parameters of the core samples

\begin{tabular}{lllll}
\hline Samples & Diameter /cm & Length /cm & Porosity /\% & $\begin{array}{l}\text { Permeability } \\
/ \times 10^{-3} \mu \mathrm{m}^{2}\end{array}$ \\
\hline DBC81 & 2.51 & 3.52 & 9.50 & 0.26 \\
DBC82 & 2.51 & 3.40 & 6.71 & 0.24 \\
WQC91 & 2.50 & 3.50 & 14.56 & 0.31 \\
WQC92 & 2.50 & 3.35 & 13.86 & 0.11 \\
\hline
\end{tabular}

The simulated stratum water was a $\mathrm{CaCl}_{2}$ solution with a total dissolved solid concentration of $25,000 \mathrm{mg} / \mathrm{L}$. The simulated oil was a mixture of crude oil and kerosene at a ratio of 1:3. The viscosity and density of the simulated oil were $6.55 \mathrm{mPa} \cdot \mathrm{s}$ and $0.81 \mathrm{~g} / \mathrm{cm}^{3}$, respectively. The spontaneous imbibition fluid and the displacement fluid were $\mathrm{MnCl}_{2}$ solutions with a total dissolved solid concentration of $25,000 \mathrm{mg} / \mathrm{L} ; \mathrm{Mn}^{2+}$ could weaken the relaxation time of hydrogen atoms in the water, effectively screening the hydrogen signal in the simulated stratum water. Using a temperature of $55{ }^{\circ} \mathrm{C}$ and core analysis practices (SY/T 5336-2006, Chinese standard), the oil displacement measurements were conducted. NMR measurements were performed according to the specifications for the measurement of the rock NMR parameters in the laboratory (SY/T 6490-2016, Chinese standard). The measurement procedures were as follows:

1. The samples were washed oil with the dissolvent of benzene and alcohol (at a ratio of 3:1) for 10 days. Subsequently, the samples were dried at $105{ }^{\circ} \mathrm{C}$ for $48 \mathrm{~h}$, and the mass and permeability of the samples were measured.

2. The core samples were placed in an LDY-150 displacement seepage instrument at a flow rate of $0.05 \mathrm{~mL} / \mathrm{min}$. The samples were saturated with the simulated stratum water, and the core samples were weighed to determine their porosity.

3. At a flow rate of $0.05 \mathrm{~mL} / \mathrm{min}$, the $\mathrm{MnCl}_{2}$ solution was used to displace the simulated stratum water; when the volume of the liquid from the point of exit reached to 3-4 times the pore volume, it was considered that the simulated stratum water was evidently displaced.

4. At a flow rate of $0.05 \mathrm{~mL} / \mathrm{min}$, the simulated oil was used to displace the $\mathrm{MnCl}_{2}$ solution; when the liquid from the point was saturated with simulated oil, the primary oil and water distribution was considered to be established, and the samples subsequently subjected to NMR measurements.

5. The displacement measurements of the core samples were carried out at a rate of $0.02 \mathrm{~mL} / \mathrm{min}$, and the NMR measurements were performed again after the displacement measurements; following that, the procedures (1) to (4) were repeated, and the displacement measurements at a rate of $0.04 \mathrm{~mL} / \mathrm{min}, 0.06 \mathrm{~mL} / \mathrm{min}$ and $0.08 \mathrm{~mL} / \mathrm{min}$ were carried out.

6. Procedures (1) to (4) were repeated, the core samples were set into the $\mathrm{MnCl}_{2}$ solution, and the NMR $T_{2}$ measurements were performed at various spontaneous imbibition times.

7. When the spontaneous imbibition measurements were completed, a reasonable displacement rate was exerted, and the NMR $T_{2}$ measurements were performed again.

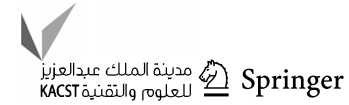




\section{NMR theory}

NMR relaxation occurs when material with a magnetic nucleus enters a magnetic field. The NMR relaxation time in the low-field NMR measurements can be divided into surface, bulk and diffusion relaxation times (Kenyon et al. 1988; Howard et al. 1993). The NMR relaxation time can be expressed as follows:

$\frac{1}{T_{2}}=\frac{1}{T_{2 B}}+\frac{1}{T_{2 S}}+\frac{1}{T_{2 D}}$

where $T_{2 \mathrm{~B}}$ is the bulk relaxation time, $T_{2 \mathrm{~S}}$ is the surface relaxation time, and $T_{2 \mathrm{D}}$ is the diffusion relaxation time, respectively. Typically, $T_{2 \mathrm{~B}}$ and $T_{2 \mathrm{D}}$ can be ignored in porous media, and $T_{2}$ can be approximately replaced with $T_{2 \mathrm{~S}}$ (Guo and Kantzas 2009).

$\frac{1}{T_{2}}=\frac{1}{T_{2 S}}$

Surface relaxation commonly occurs at the interface of a solid and a liquid, and it is related to the pore structure of the porous medium. $T_{2 \mathrm{~S}}$ can be expressed as follows:

$\frac{1}{T_{2 S}}=\rho\left(\frac{S}{V}\right)_{\text {pore }}=\frac{c}{r}$

where $\rho$ is the relaxation rate of the particle surface, $r$ is the pore aperture $(\mu \mathrm{m})$; and $c$ is a nondimensional parameter related to the pore structure.

Evidently, the different transverse relaxation times acquired from the NMR measurements could approximate the pore aperture in porous medium.

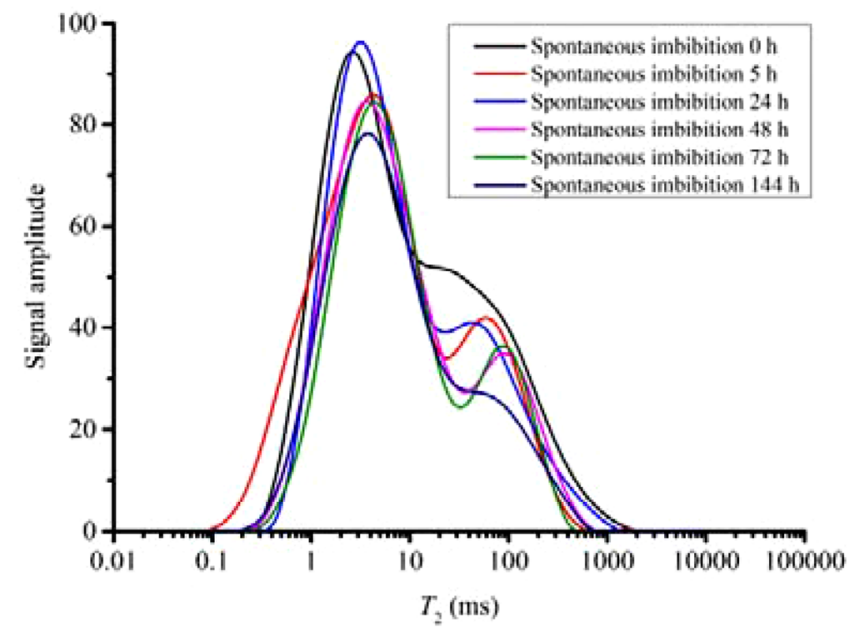

(a) DBC81

\section{Fractal theory}

Fractal theory can effectively evaluate the irregularity degree and self-similarity of pores (Mandelbrot 1979; Pfeifer and Avnir 1983; Yang et al. 2016; Garcia-Gutierrez et al. 2017). A larger fractal dimension indicates a complex pore structure and the strong heterogeneity of a reservoir (Zhang et al. 2017; Tang et al. 2019).

According to fractal theory (Mandelbrot et al. 1984), the number of pores with apertures greater than $r$ can be expressed as follows:

$N_{r}=\int_{r}^{r_{\max }} f(r) d r=A r^{-D}$

where $N_{\mathrm{r}}$ is the number of pores with apertures greater than $r ; r$ is the pore aperture $(\mu \mathrm{m}) ; r_{\max }$ is the maximum aperture of the pore $(\mu \mathrm{m}) ; A$ is the fractal factor, $f(r)$ is the density function of the pore aperture (\%); and $D$ is the fractal dimension. Using the first derivation of Eq. (4),

$f(r)=\frac{d N_{r}}{d r}=A^{\prime} r^{-D-1}$

where $A^{\prime}$ is a proportionality constant. The cumulative volume of pores with apertures below $r$ can expressed as follows:

$V_{r}=\int_{r_{s}}^{r} f(r) A r^{3} d r=A^{\prime \prime}\left(r^{3-D}-r_{\min }^{3-D}\right)$

where $V_{r}$ is the cumulative volume of pores with an aperture below $r\left(\mathrm{~cm}^{3}\right), A^{\prime \prime}$ is a constant of proportionality, and $r_{\min }$ is the minim aperture of the pore $(\mu \mathrm{m})$.

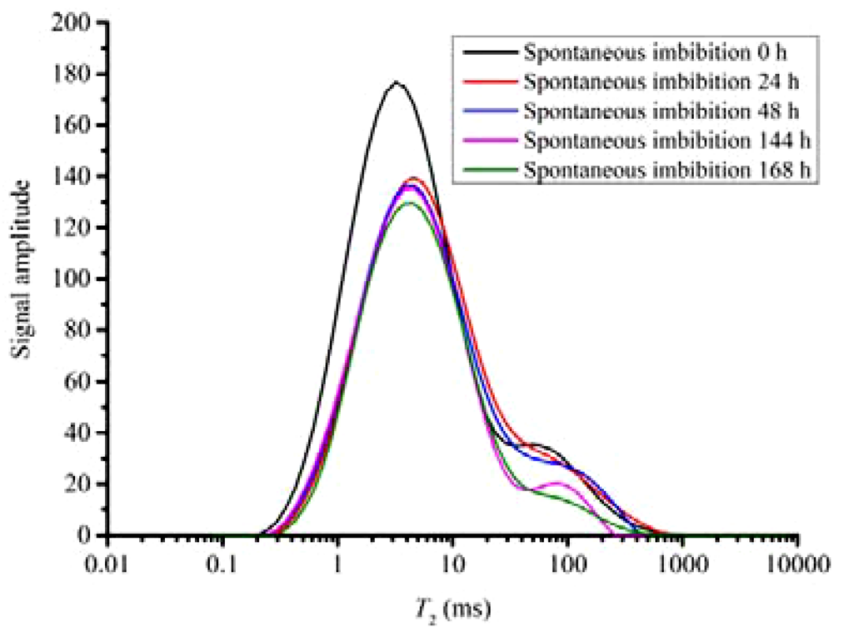

(b) WQC91

Fig. 1 NMR $T_{2}$ spectra of residual oil at various SI times 


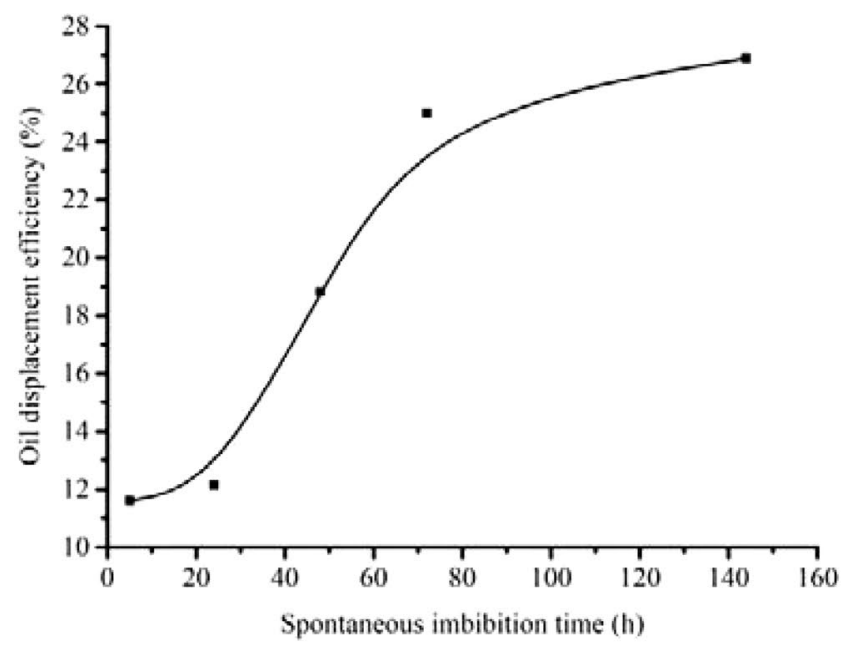

(a) DBC81

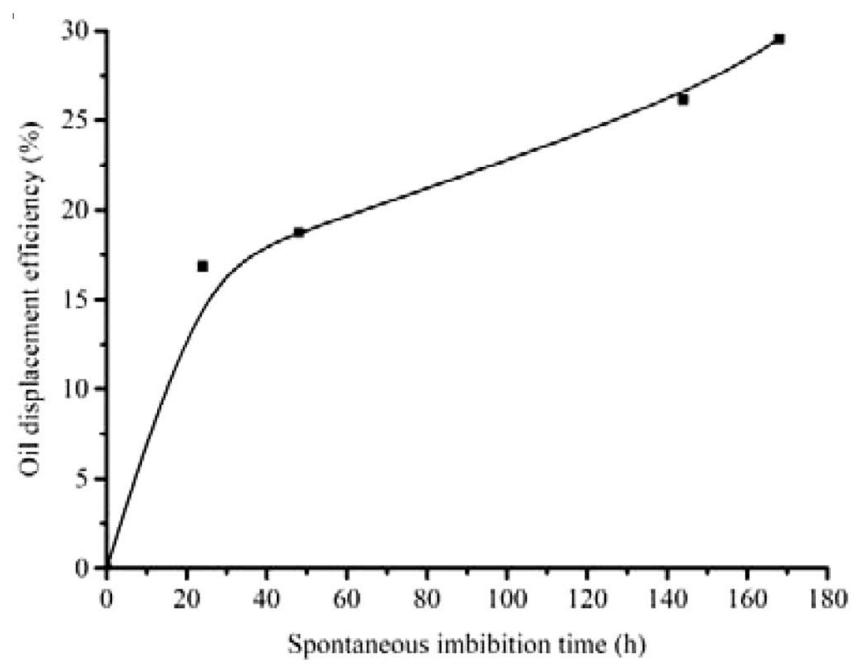

(b) WQC91

Fig. 2 Oil displacement efficiencies at various spontaneous imbibition times

The total pore volume of the pores could be presented as follows:

$V_{t}=A^{\prime \prime}\left(r_{\max }^{3-D}-r_{\min }^{3-D}\right)$

where $V_{t}$ is the total pore volume $\left(\mu \mathrm{m}^{3}\right)$. Then, the cumulative volume fraction for pores with apertures below $r$ can be expressed as follows:

$S_{v}=\frac{V_{r}}{V_{t}}=\frac{r^{3-D}-r_{\min }^{3-D}}{r_{\max }^{3-D}-r_{\min }^{3-D}}$

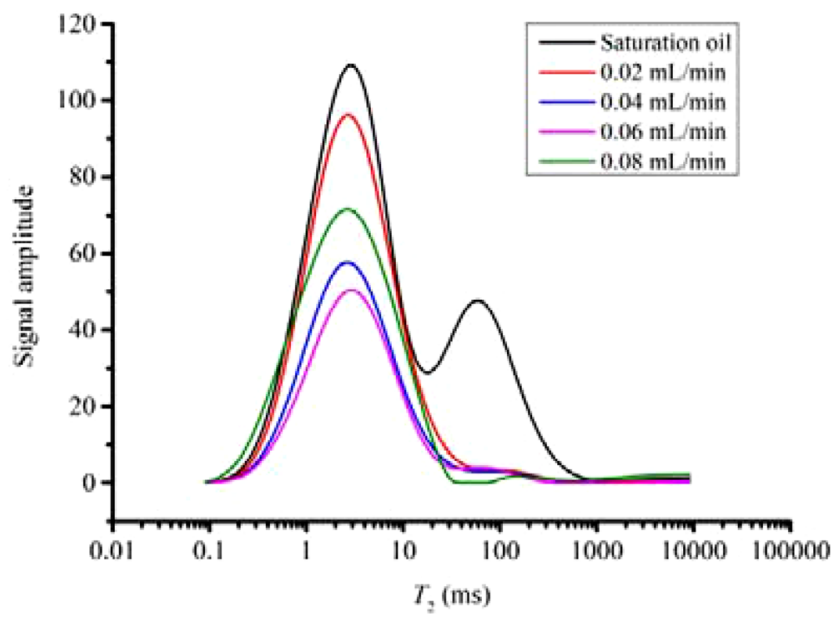

(a) $\mathrm{DBC} 82$
If $r_{\min }$ is far lower than $r_{\text {max }}$, then $r_{\text {min }}$ can be ignored; and Eq. (8) can be simplified as follows:

$S_{v}=\left(\frac{r}{r_{\max }}\right)^{3-D}$

Using Eq. (3), Eq. (9) can be modified as follows:

$S_{v}=\left(\frac{T_{2 \max }}{T_{2}}\right)^{D-3}$

By logarithmically transforming Eq. (10), we obtain

$\lg \left(S_{v}\right)=(3-D) \lg T_{2}+(D-3) \lg T_{2 \max }$

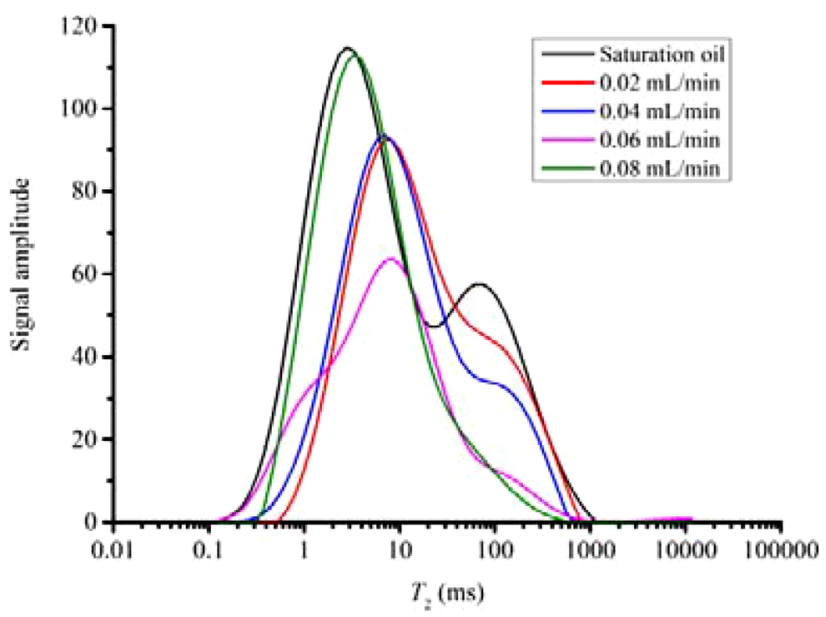

(b) WQC92

Fig. 3 NMR $T_{2}$ spectra of residual oil at various water flooding rates 


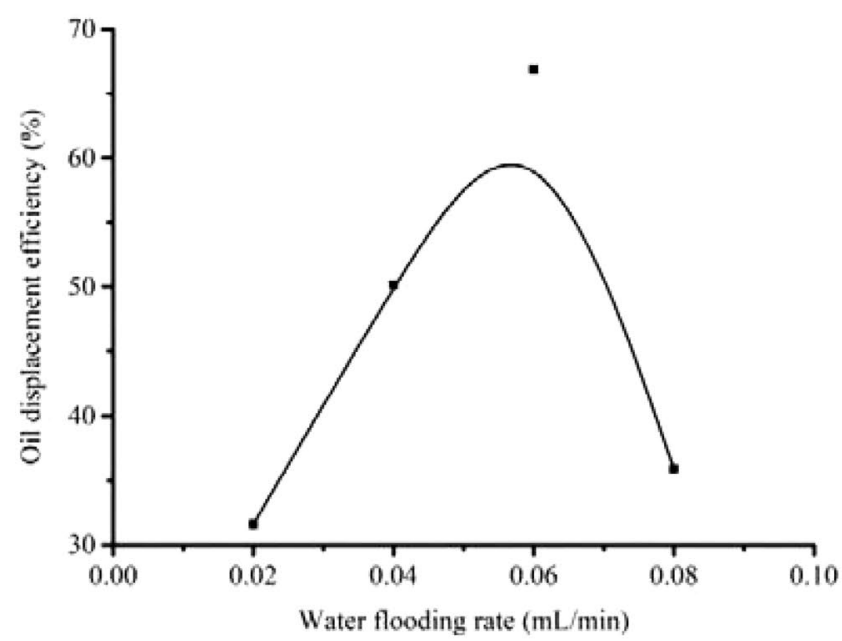

(a) $\mathrm{DBC} 82$

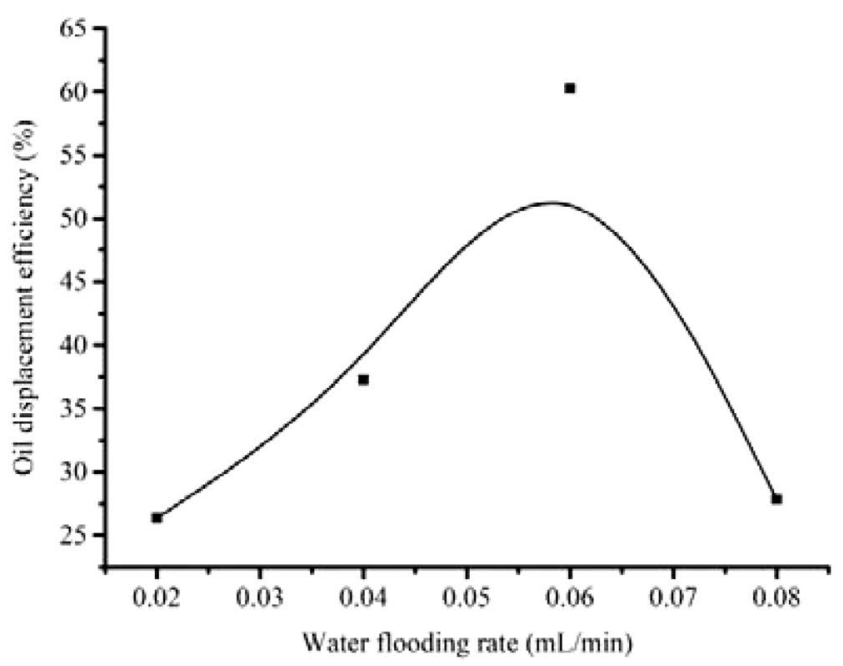

(b) WQC92

Fig. 4 Oil displacement efficiencies of tight core samples at various water flooding rates

Currently, Eq. (11) has been widely used to evaluate the fractal characteristics of pores in tight sandstones (Al-Mahrooqi et al. 2003; Shao et al. 2017).

\section{Results}

\section{Spontaneous imbibition displacement of tight oil}

Evidently, the residual oil of the NMR $T_{2}$ spectrum for DBC81 and WQC91 samples has bimodal features before the spontaneous imbibition (SI) displacement. With continued SI displacement, the bimodal peaks tended to decrease, indicating that the residual oil in the cores began to discharge (Fig. 1). The oil displacement efficiency can be calculated with the decrease in the peak area at various spontaneous imbibitions times with Eq. (12). Owing to the continuous discharge of tight oil from the pores, the oil displacement efficiency increased (Fig. 2). The SI oil displacement efficiency was low during the initial $24 \mathrm{~h}$; subsequently, it increased sharply and tended to lower after a total SI time of $144 \mathrm{~h}$, when the total oil displacement efficiency reached $26.91 \%$. For the WQC91 core sample, the oil displacement efficiency increased rapidly to $16.86 \%$ at

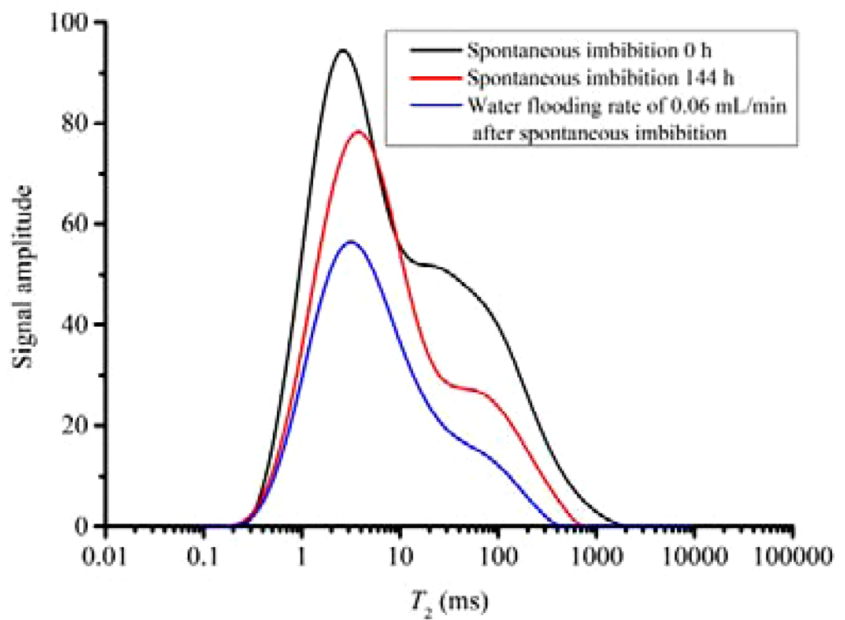

(a) $\mathrm{DBC} 81$

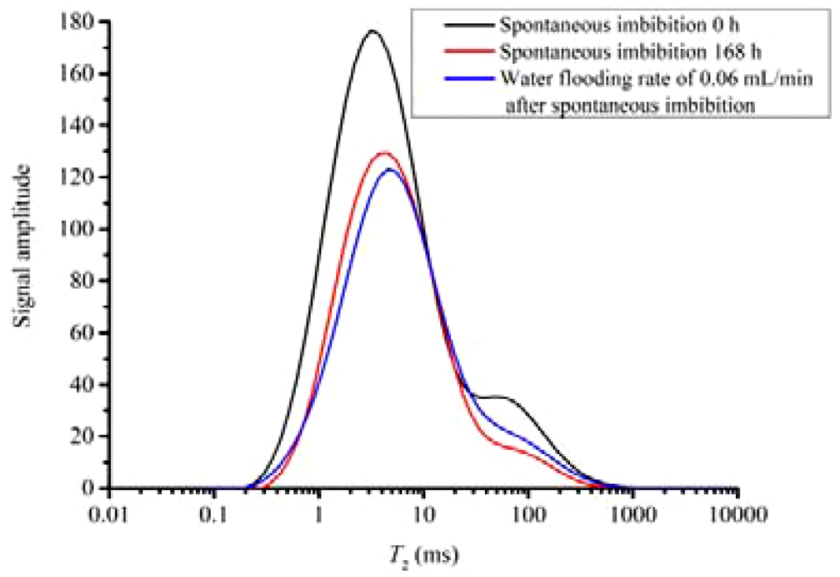

(b) WQC91

Fig. 5 NMR $T_{2}$ spectrum of residual oil for core samples under a water flooding rate of $0.06 \mathrm{~mL} / \mathrm{min}$ after SI 


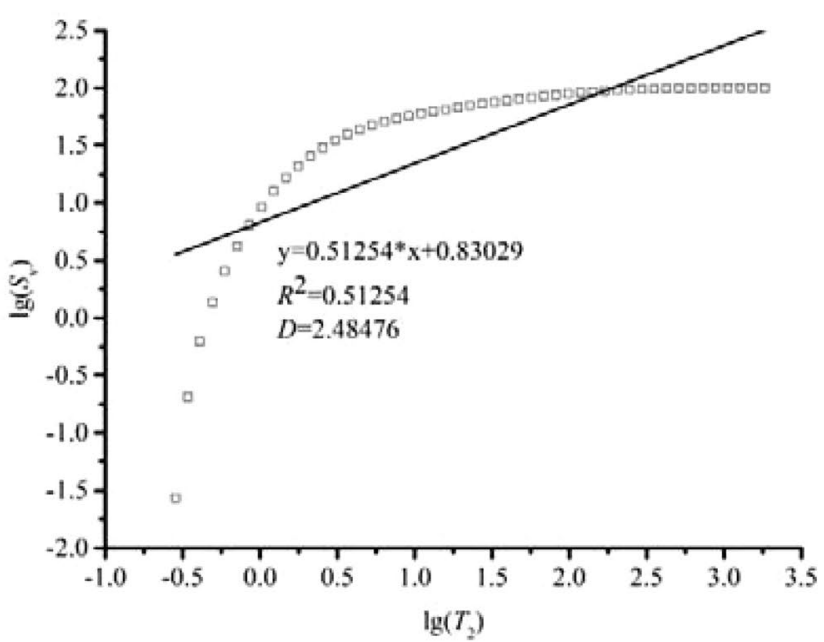

(a) DBC81

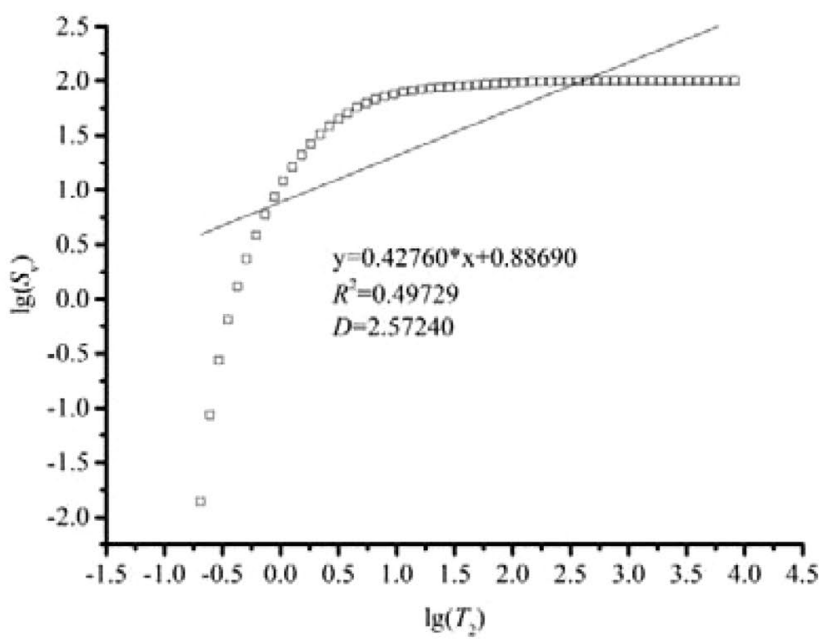

(c) WQC91

Fig. 6 Relationships between the $\lg \left(T_{2}\right)$ and $\lg \left(S_{\mathrm{v}}\right)$ for core samples

an SI time of $24 \mathrm{~h}$, followed by a slow increase. The total SI oil displacement efficiency was approximately $29.56 \%$. Although the pore structures of DBC81 and WQC91 are similar, the various pore structure complexities may lead to different oil displacement characteristics (Fig. 2).

oil displacement efficiency $=\frac{A-A_{i}}{A}$

where $A$ is the peak area of samples at the spontaneous imbibition time of $0 \mathrm{~h}$; and $A_{i}$ is the corresponding peak area of samples at other spontaneous imbibition times.

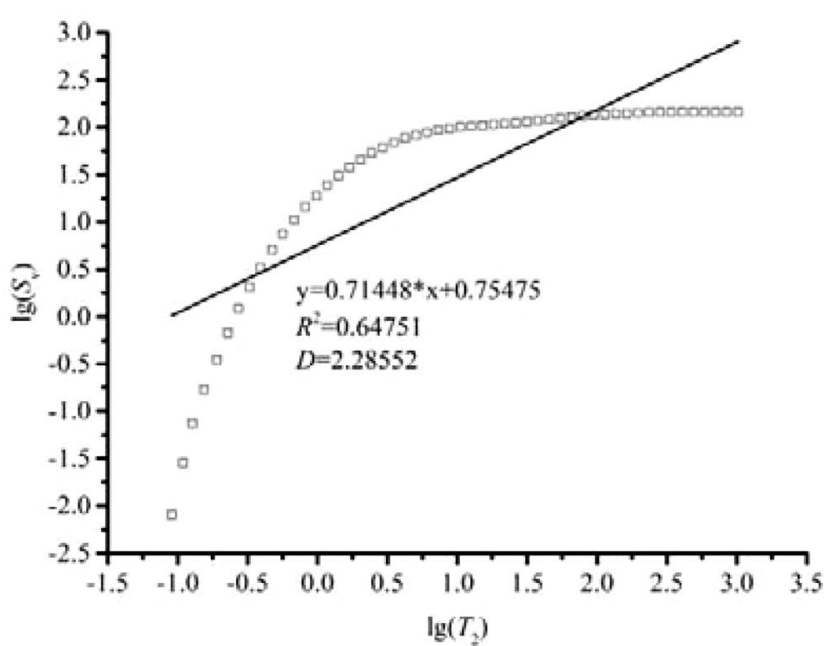

(b) $\mathrm{DBC} 82$

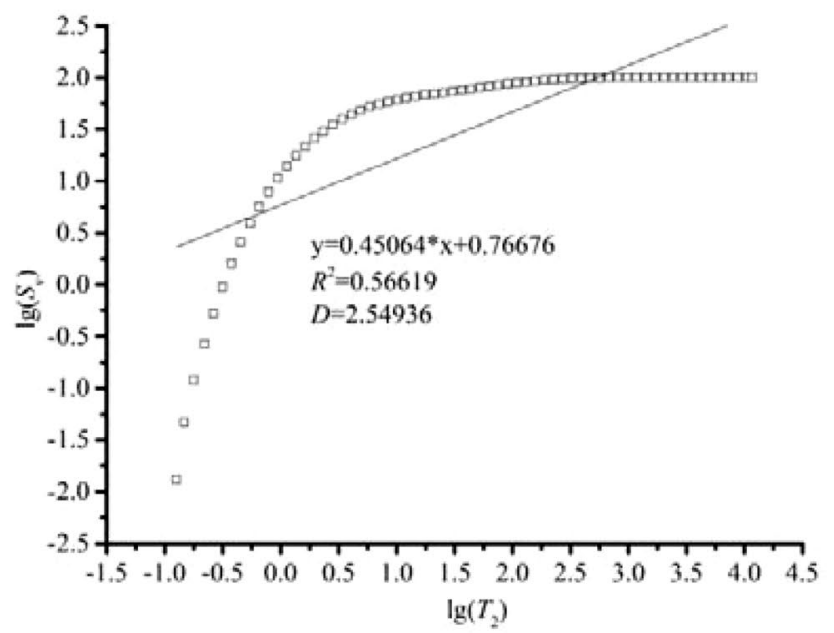

(d) WQC92

\section{Displacement of tight oil at various water flooding rates}

At a certain water flooding rate, the tight oil in the reservoir can be rapidly discharged. For the same tight oil reservoir, various water flooding rates may lead to different oil displacement efficiencies. In this study, the core samples were analyzed at four different water flooding rates: 0.02, 0.04, 0.06 and $0.08 \mathrm{~mL} / \mathrm{min}$.

The NMR $T_{2}$ spectra of the residual oil for DBC82 and WQC92 were bimodal. At a water flooding rate of $0.02 \mathrm{~mL} /$ 


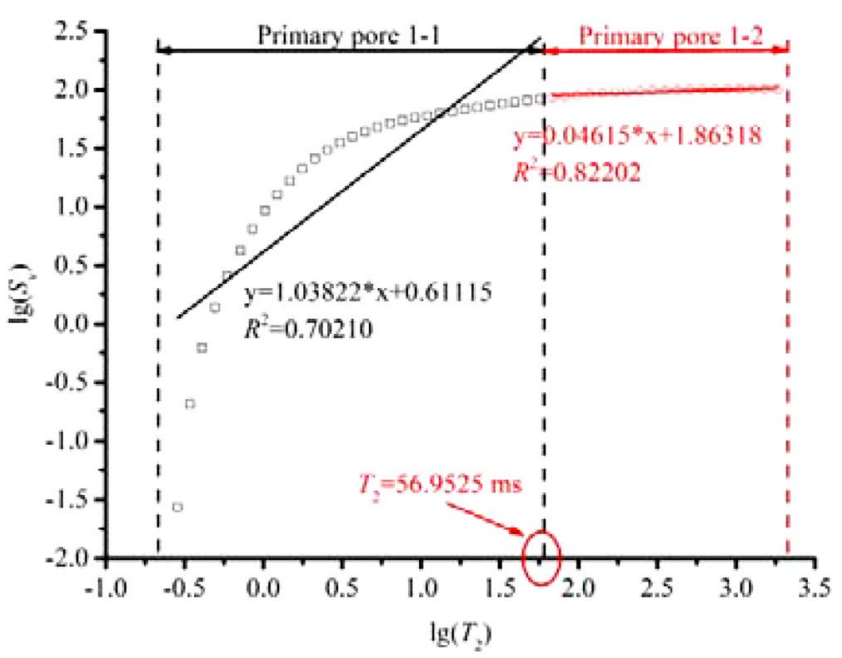

(a) DBC81

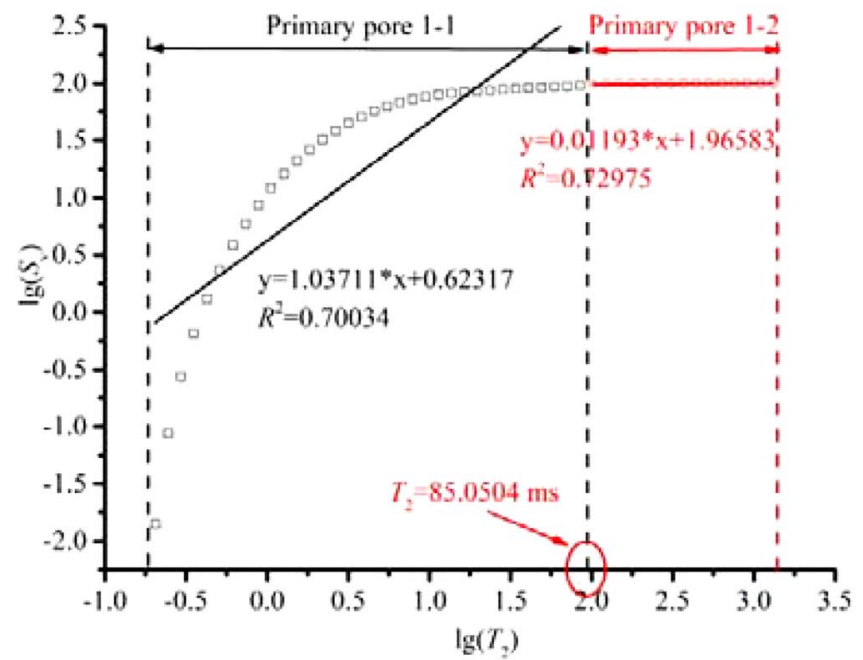

(c) WQC91

Fig. 7 Cutoff points of the primary pores for the core samples

min, the amplitude of the left and right peaks of the core samples tended to decrease, particularly that for the right peak, indicating that the tight oil in the greater pores was discharged. When the water flooding rates increased, the left peak features decreased first and then increased, whereas the amplitude of the right peak tended to continuously decrease, and the degree of decrease in the left peak reached a maximum when the water flooding rate was $0.06 \mathrm{~mL} / \mathrm{min}$ (Fig. 3).

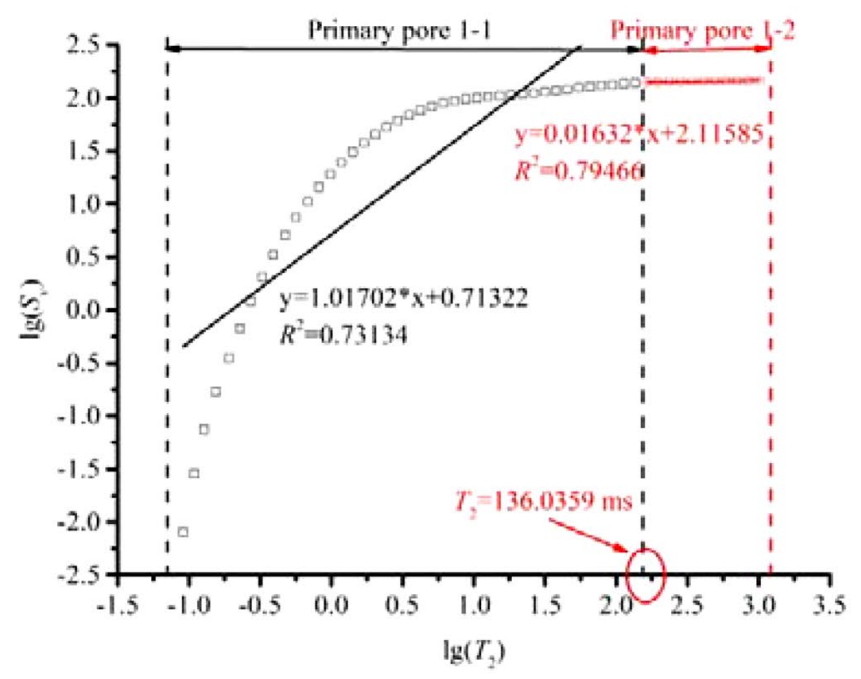

(b) $\mathrm{DBC} 82$

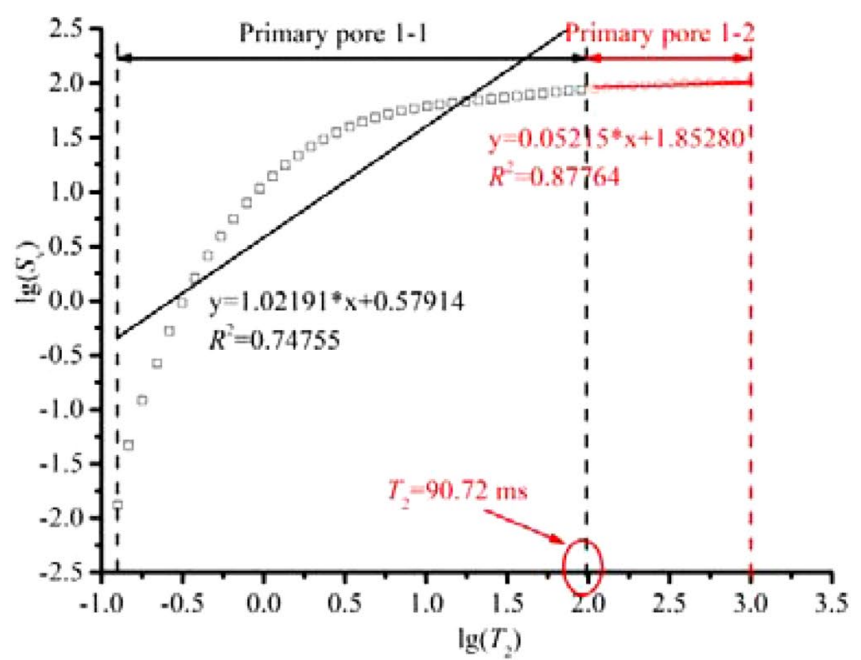

(d) WQC92

These results indicate that a continuous increase in the water flooding rates may not achieve the maximum oil displacement efficiency and that a reasonable water flooding rate would be optimal. The oil displacement efficiency at various water flooding rates had an inverse "V"-type shape (Fig. 4). The oil displacement efficiencies were $66.88 \%$ and $60.27 \%$ for the DBC82 and WQC92 core samples, respectively. 


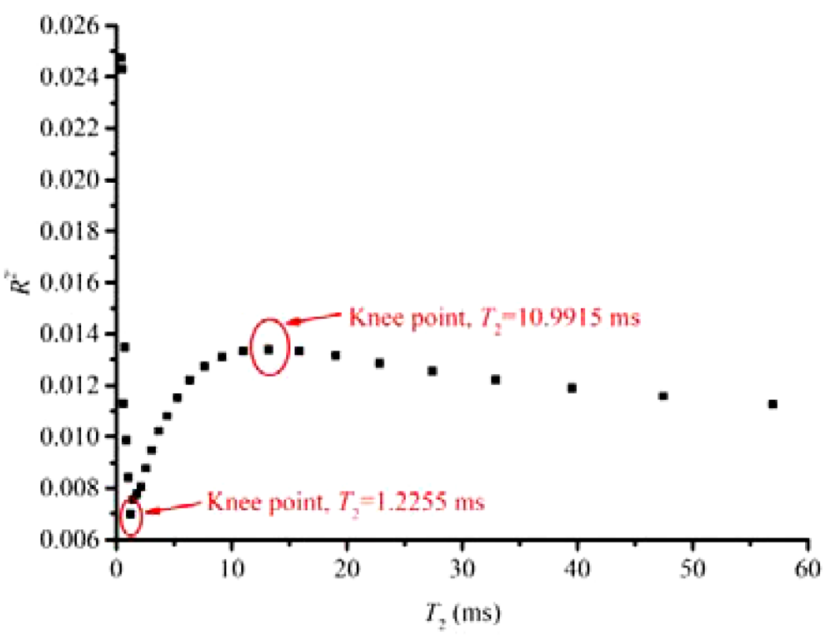

(a) DBC81 primary pore 1-1

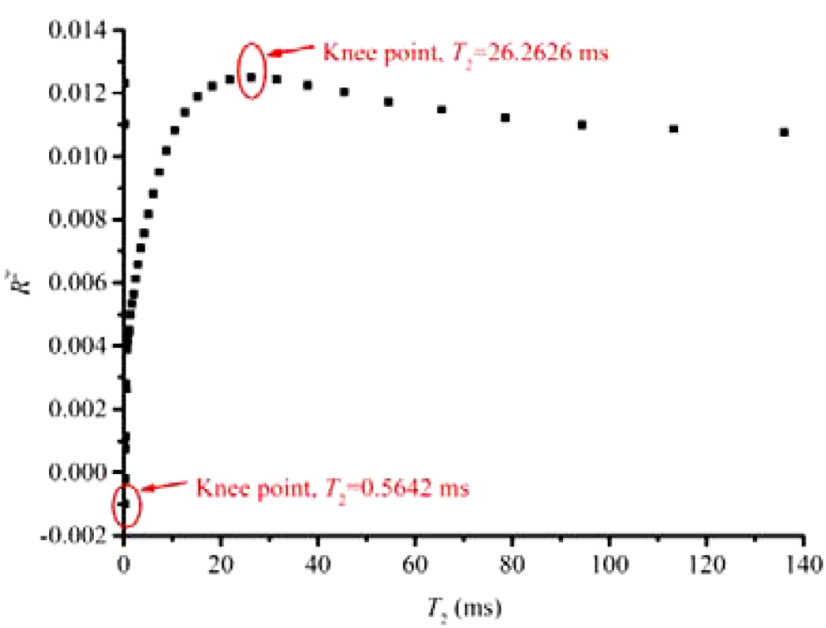

(c) DBC82 primary pore $1-1$

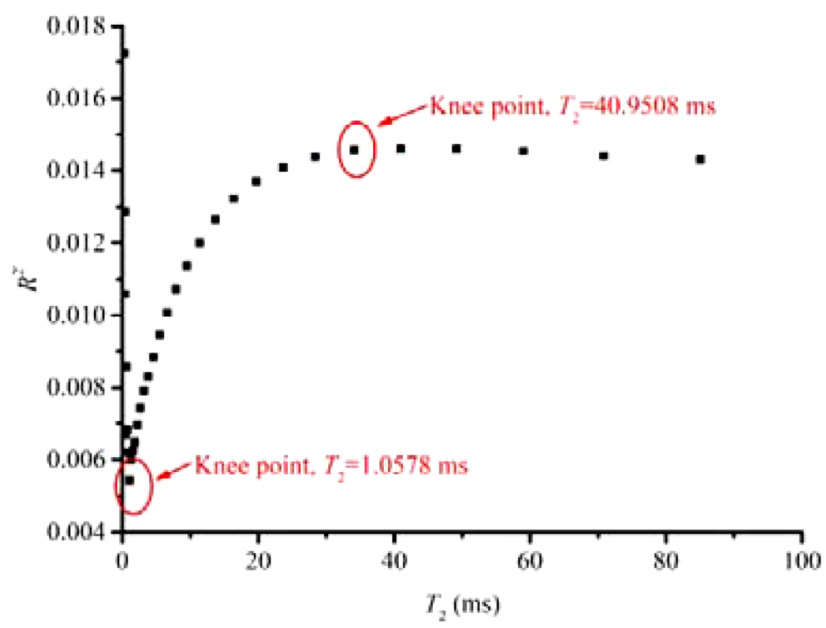

(e) WQC91 primary pore 1-1

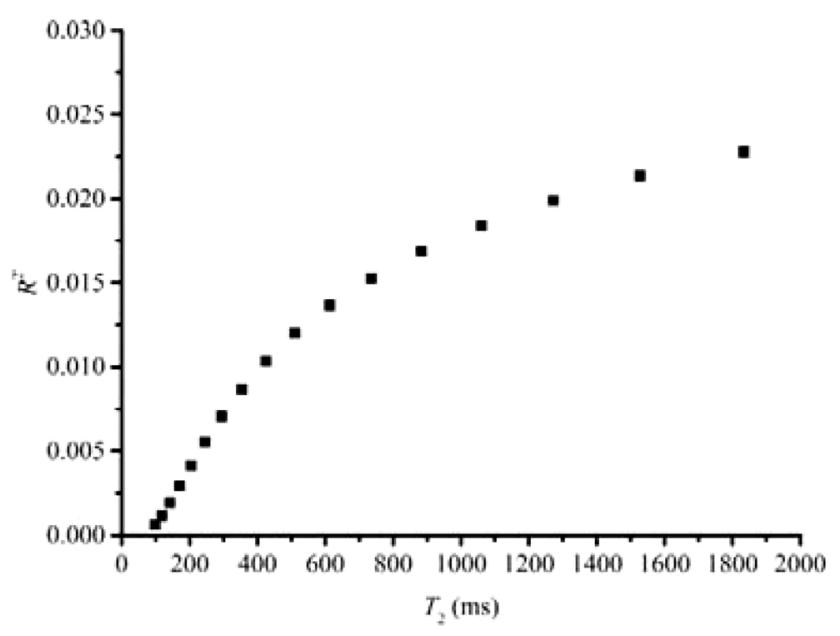

(b) DBC81 primary pore 1-2

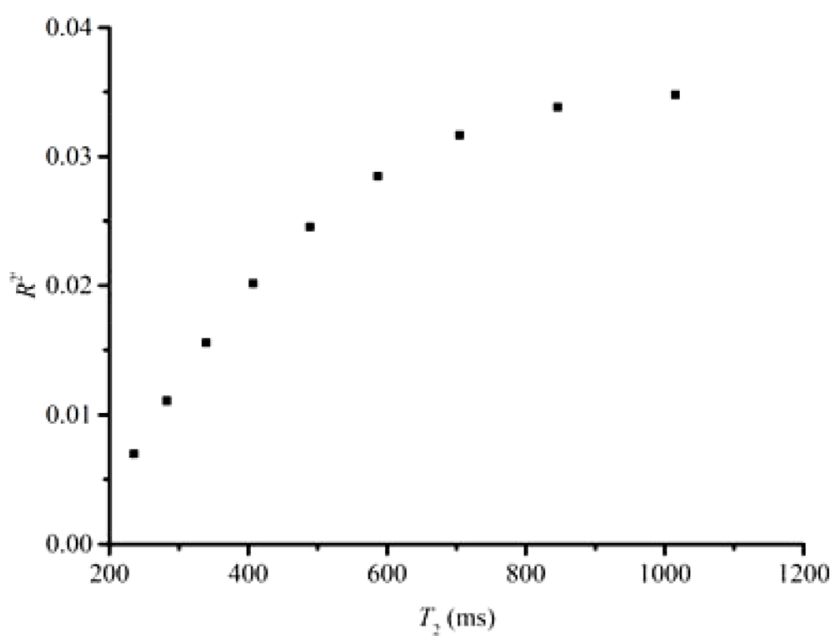

(d) DBC82 primary pore $1-2$

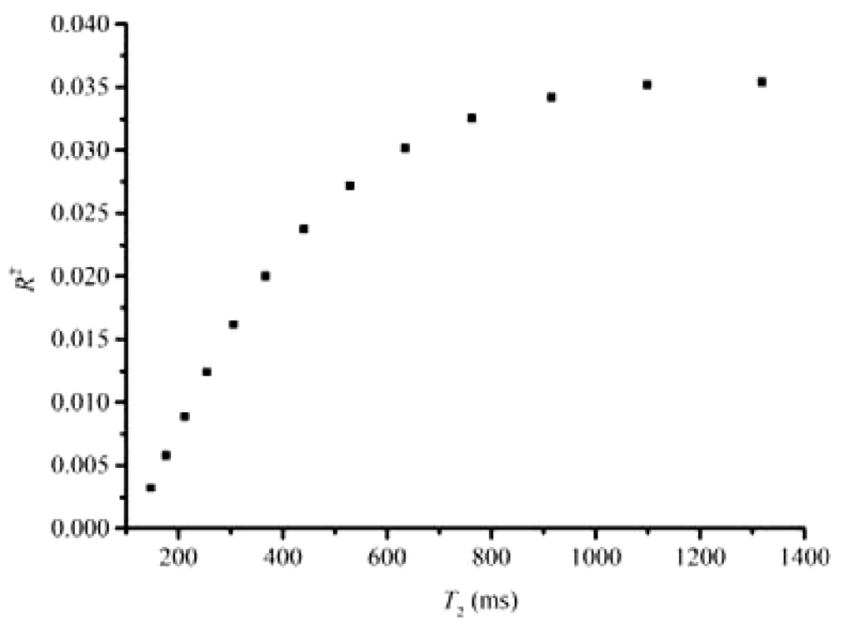

(f) WQC91 primary pore 1-2

Fig. 8 Cutoff points of the secondary pores for core samples 


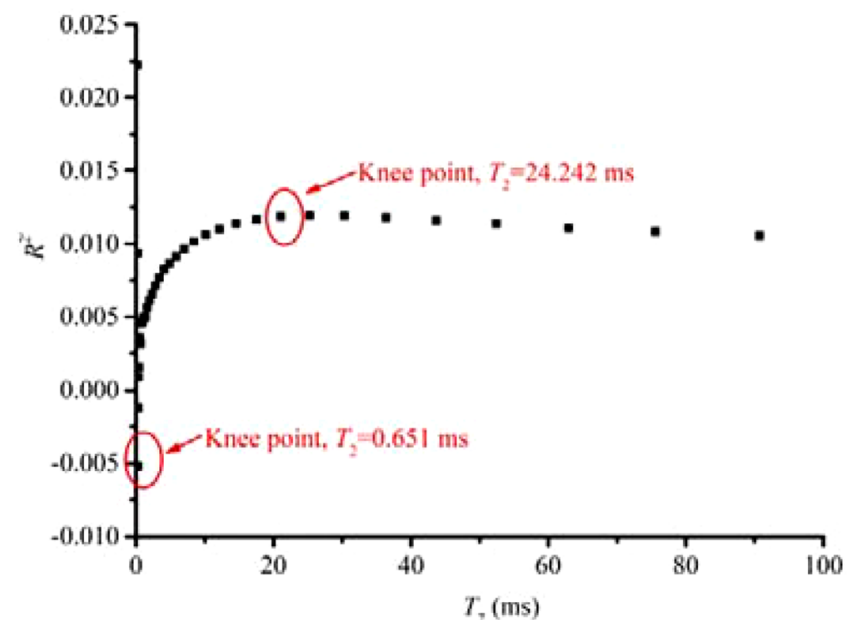

(g) WQC92 primary pore 1-1

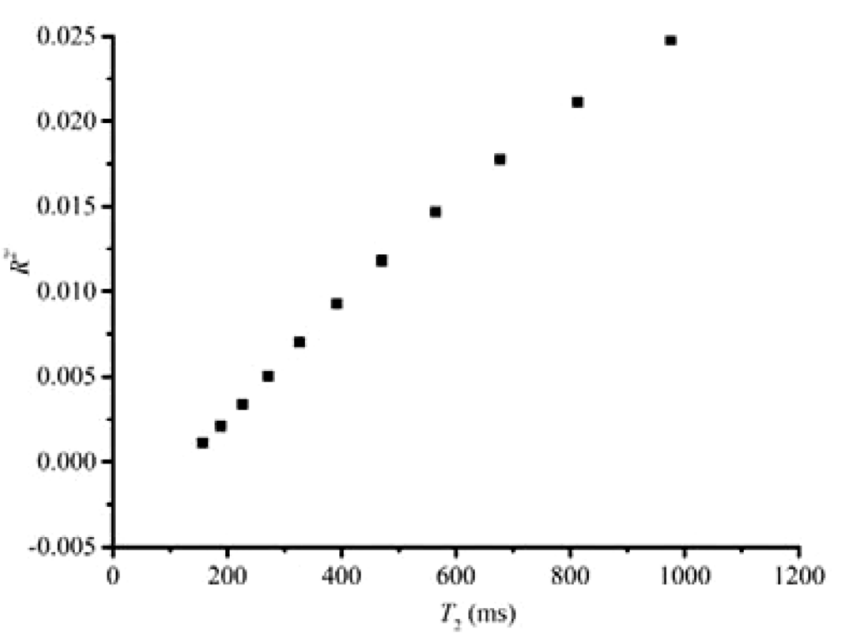

(h) WQC92 primary pore 1-2

Fig. 8 (continued)

\section{Water flooding after SI}

After the SI, there was still a certain amount of residual oil in the core samples, and a reasonable water flooding rate of $0.06 \mathrm{~mL} / \mathrm{min}$ can be ensured; thus, the water flooding rate of $0.06 \mathrm{~mL} / \mathrm{min}$ was used for the DBC81 and WQC91 core samples after the SI. Evidently, the amplitude of the left and right peaks significantly decreases when the water flooding is launched after the SI (Fig. 5a), and the oil displacement efficiency finally reaches $50.02 \%$. For WQC91, there was still a certain decrease in the amplitude of both the left and right peaks, while it was unapparent, and the oil displacement efficiency reached to approximately $30.35 \%$.

\section{Discussion}

\section{Pore type classification method}

The pores in a porous medium features fractal characteristics, and the fractal method can distinguish the various pore types. In this study, a new pore type classification method called the progressively refined pore classification method is proposed. Before the fractal dimensions were calculated, some deviation data were deleted, particularly the $T_{2}$ obtained in response to a signal with no amplitude.
Evidently, the core samples featured fractal characteristics (Fig. 6). Typically, the surface of a pore was irregular, and a knee point existed for the fractal curves of the porous medium (Wang et al. 2020). The relationship between $\lg \left(T_{2}\right)$ and $\lg \left(S_{\mathrm{v}}\right)$ can be further divided into two parts.

According to Eq. (11), the relationship between the transversal relaxation time and cumulative signal amplitude fraction can be expressed as follows:

$\lg \left(S_{v i}\right)=\left(3-D_{i}\right) \lg \left(T_{2 i}\right)+\left(D_{i}-3\right) \lg \left(T_{2 \max }\right)$

where $S_{\mathrm{vi}}$ is the cumulative signal amplitude fraction for $T_{2 \mathrm{i}}(\%) ; D_{\mathrm{i}}$ is the fractal dimension for $T_{2 \mathrm{i}}$; and $T_{2 \max }$ is the maximum transverse relaxation time (ms).

Using Eq. (13), the fractal dimensions at various transversal relaxation times could be acquired. The porous medium features fractal characteristics, and a fractal dimension of 2 was selected as the cutoff point (Guo et al. 2019). Afterward, the cutoff point of $T_{2}$ for the different core samples could be acquired (Fig. 7).

When the cutoff points of the primary pores were acquired, the fractal dimensions $(D)$ and corresponding correlation coefficient $\left(R^{2}\right)$ were also gained. $R^{2}$ reflects the deviation degree of the data, and a significant change in $R^{2}$ implies that the newly added data are significantly different from the former data, and this difference can be calculated as follows: 


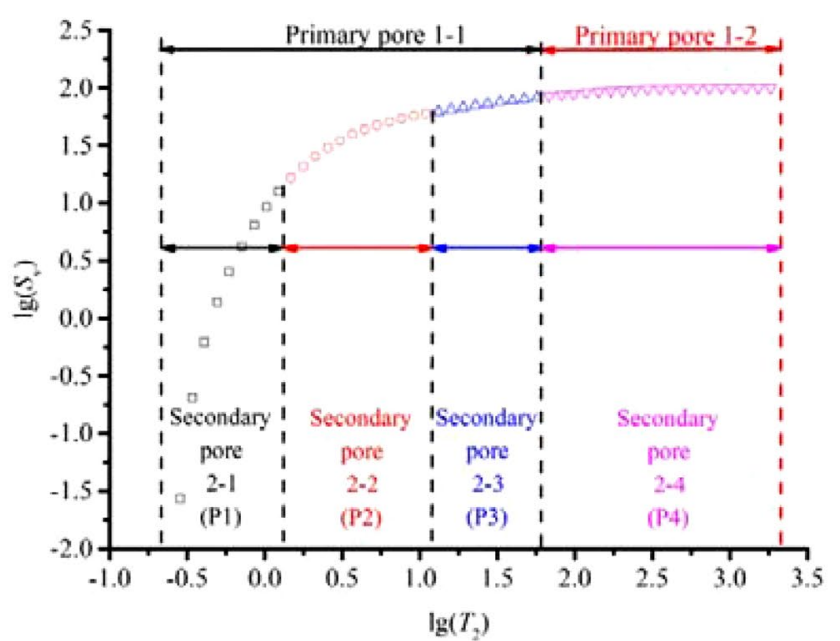

(a) DBC81

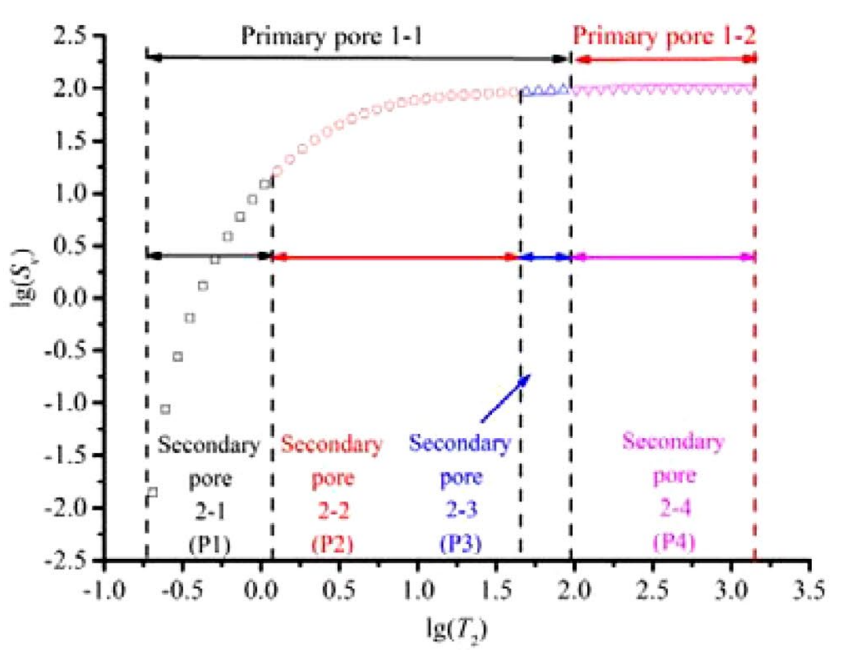

(c) WQC91

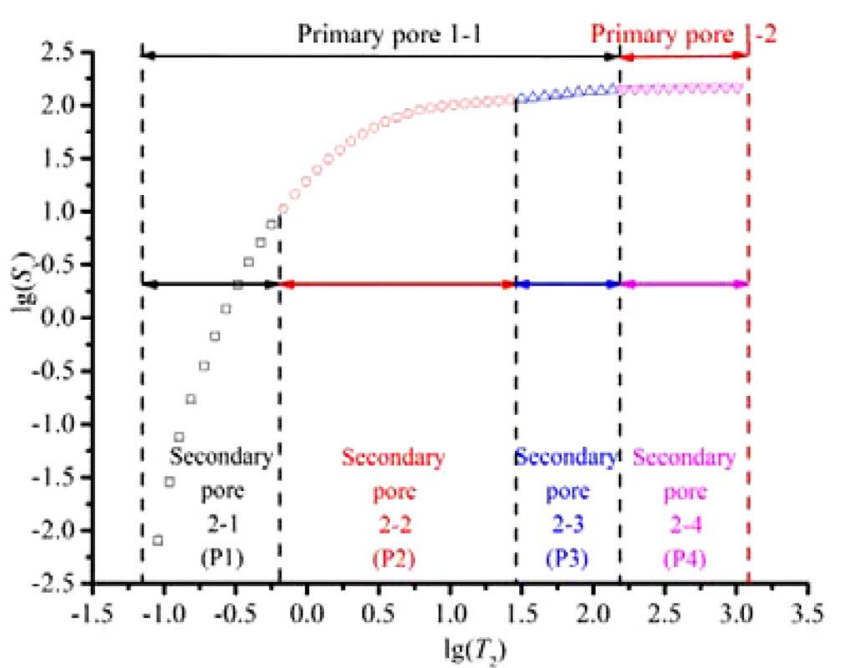

(b) $\mathrm{DBC} 82$

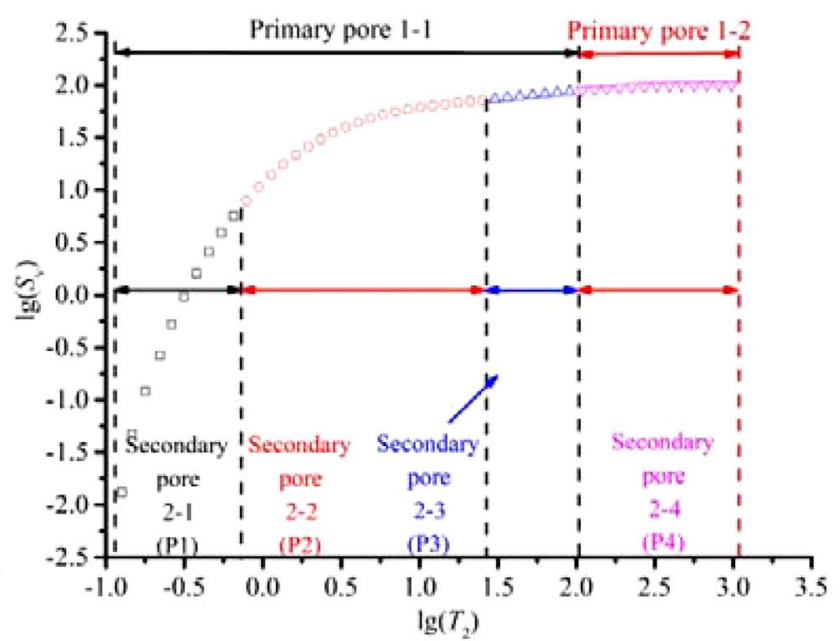

(d) WQC92

Fig. 9 Pore types for the core samples using the progressively refined pore classification method

$R^{2^{\prime}}=\frac{R_{i+1}^{2}-R_{i}^{2}}{R_{i}^{2}}$

where $R_{i}^{2}$ is the correlation coefficient of the relationship between $\lg \left(T_{2 \mathrm{i}}\right)$ and $\lg \left(S_{\mathrm{vi}}\right)$ under $T_{2 \mathrm{i}}$, and $R_{i+1}^{2}$ is the correlation coefficient of the relationship between $\lg \left(T_{2 \mathrm{i}+1}\right)$ and $\lg \left(S_{\mathrm{vi}+1}\right)$ under $T_{2 \mathrm{i}+1}$.

According to Eq. (14), there are two knee points for primary pore $1-1$ and no knee point for primary pore $1-2$ (Fig. 8).
Based on the primary pore classification, the secondary pores were further divided further, and the pores in the tight sandstone sample were divided into four types: secondary pore $2-1(\mathrm{P} 1)$, secondary pore $2-2(\mathrm{P} 2)$, secondary pore $2-3$ (P3), and secondary pore 2-4 (P4) (Fig. 9).

Evidently, the amplitudes of the peaks of the various pore types variably decreases after oil displacement, and all these pores were movable fluid pores (Fig. 10). The peak of the $\mathrm{P} 4$ pore featured the most significant decrease, and the $\mathrm{P} 4$ pore indicated that the fluid in it could be discharged easily. The characteristics of the $\mathrm{P} 3$ pore were similar to those of the $\mathrm{P} 4$ pore, although there was a certain amount of residual 


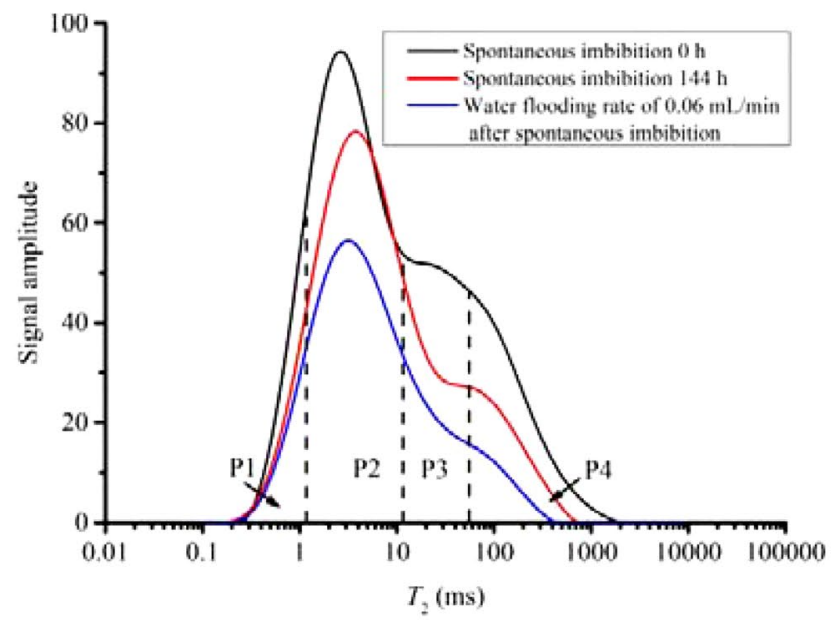

(a) $\mathrm{DBC} 81$

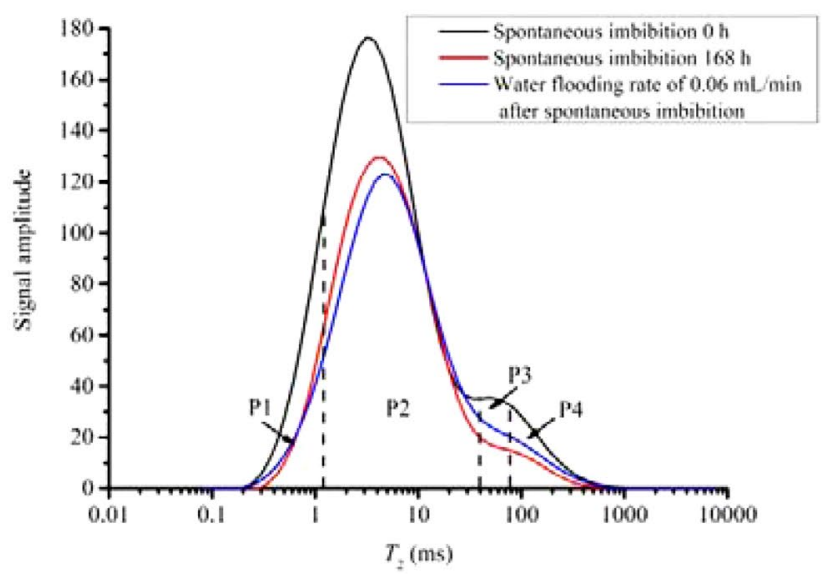

(c) WQC91

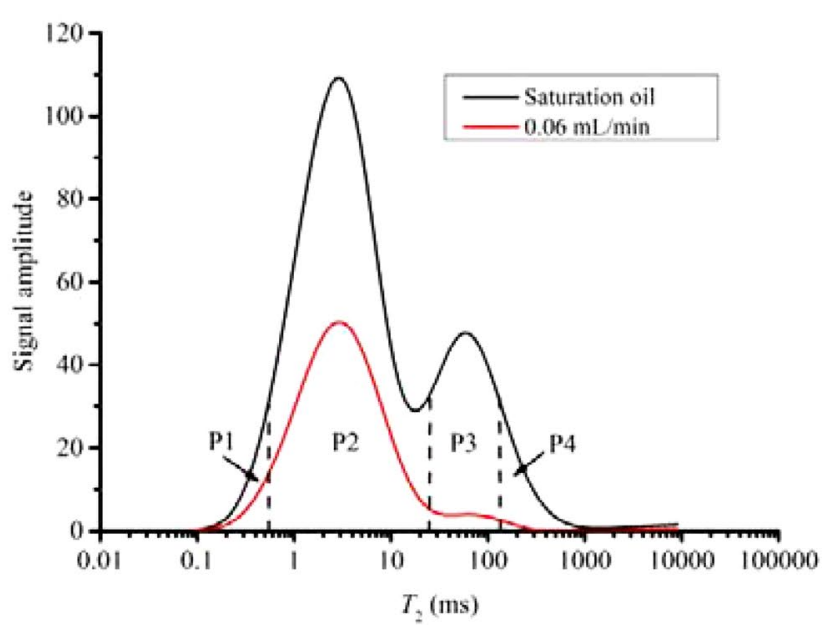

(b) $\mathrm{DBC} 82$

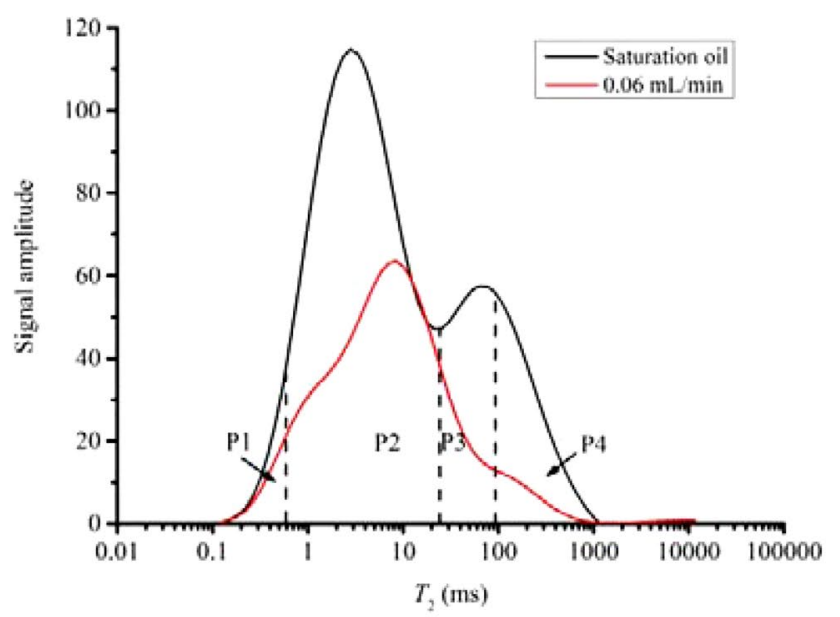

(d) WQC92

Fig. 10 Pore types and distribution in the core samples

oil in it, indicating that the fluid in the P3 pore can flow under certain condition at a lower threshold. The P2 pore was dominant in the tight core samples, and the fluids in it were partially movable. As for the P1 pore, the decrease in the peak amplitude was subtle, indicating that the fluid can hardly flow.

\section{Oil utilization degree of residual oil in various pores}

The residual oil is distributed throughout various pores, and using various displacement methods, the residual oil in various pores can be discharged further, and the oil utilization degree (OUD) of the residual oil in various pores determines the oil displacement efficiency. The OUD can be calculated using Eq. (15):

$O U D=\frac{A_{p o}-A_{p i}}{A_{p o}}$

where OUD is the degree of oil utilization of the pores (\%); $A_{p o}$ is the peak area of the pores, such as the P1, P2, P3 and $\mathrm{P} 4$ pores; and $A_{p i}$ is the corresponding peak area of the pore after the displacement.

In addition, to evaluate the OUD of the residual oil in various pores, the knee points of $T_{2}$ at the initial residual oil distribution were selected as the threshold, and the 


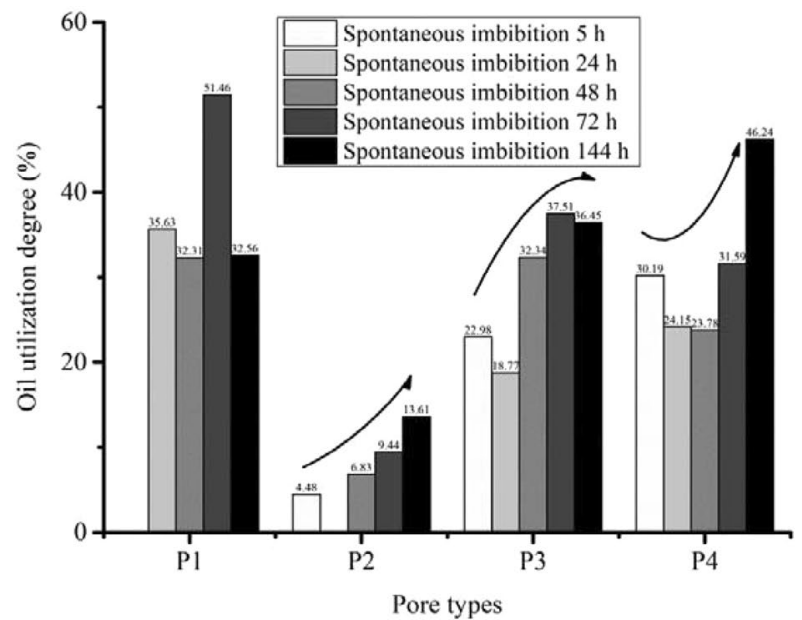

(a) $\mathrm{DBC} 81$

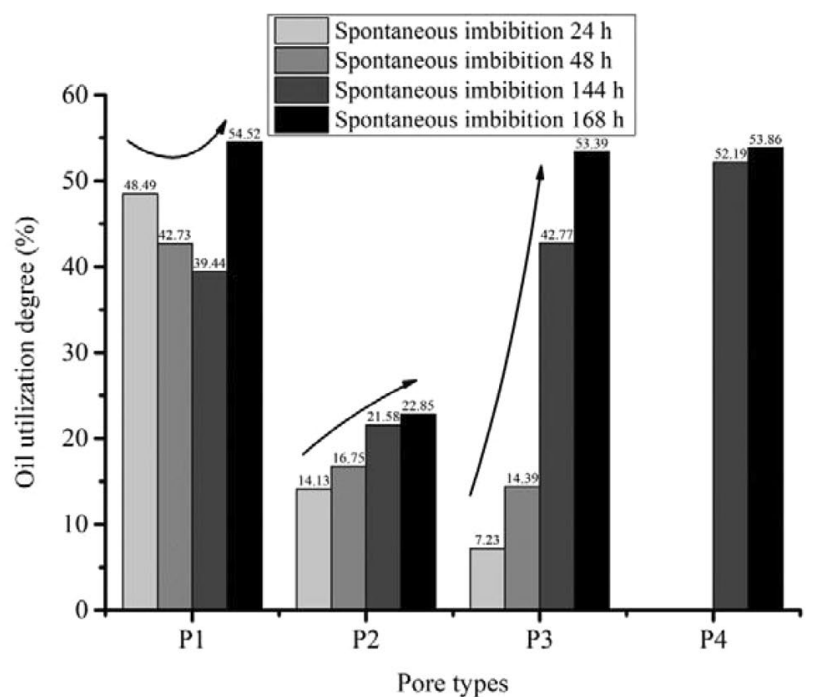

(b) WQC91

Fig. 11 OUD in various types of pores for core samples of SI measurements

OUD of residual oil in various pores was fixed for the same core sample.

\section{OUD of various pores in SI}

Figure 11 shows that the OUDs of various pores in the DBC81 and WQC91 core samples were similar. The OUD of the P1 pore features fluctuated, and there was no law during the entire SI. The OUD in the $\mathrm{P} 2$ and $\mathrm{P} 3$ pore features continuously increased with an increase in the SI time, and the increase in the oil displacement efficiency was primarily attributed to the oil volume in the P2 and

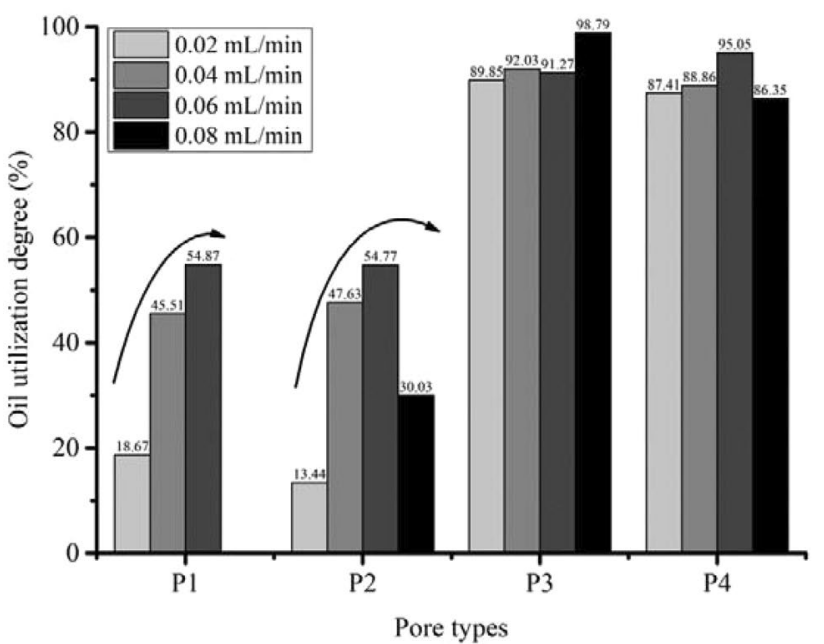

(a) $\mathrm{DBC} 81$

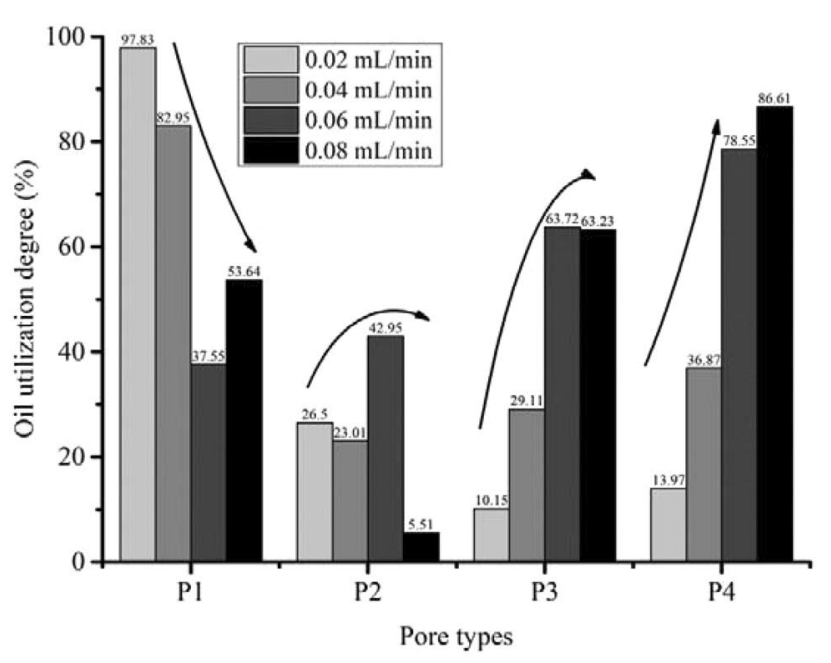

(b) WQC91

Fig. 12 OUD in various pores for the core samples at various water flooding rates

P3 pores. As shown in Fig. 10a and c, the volume of the $\mathrm{P} 2$ pores was dominant, and the OUD for the P2 pores was more essential. In addition, the OUD of the P2 and P3 pores in the DBC81 sample was lower than that of the WQC91 sample, which was primarily related to the larger pore apertures of the $\mathrm{P} 2$ and $\mathrm{P} 3$ pores in the WQC91 sample (Fig. 10a, c). The OUD of the P4 pores also fluctuated. During the initial period of the SI, the oil discharged from the $\mathrm{P} 1, \mathrm{P} 2$, and $\mathrm{P} 3$ pores migrated into the $\mathrm{P} 4$ pores; afterward, the residual oil in the $\mathrm{P} 4$ pores began to discharge rapidly, which leads to a "V"-type shaped OUD of the P4 pores for the DBC81 sample (Fig. 11a). The OUD of various pores in SI indicates that the capillary imbibition 


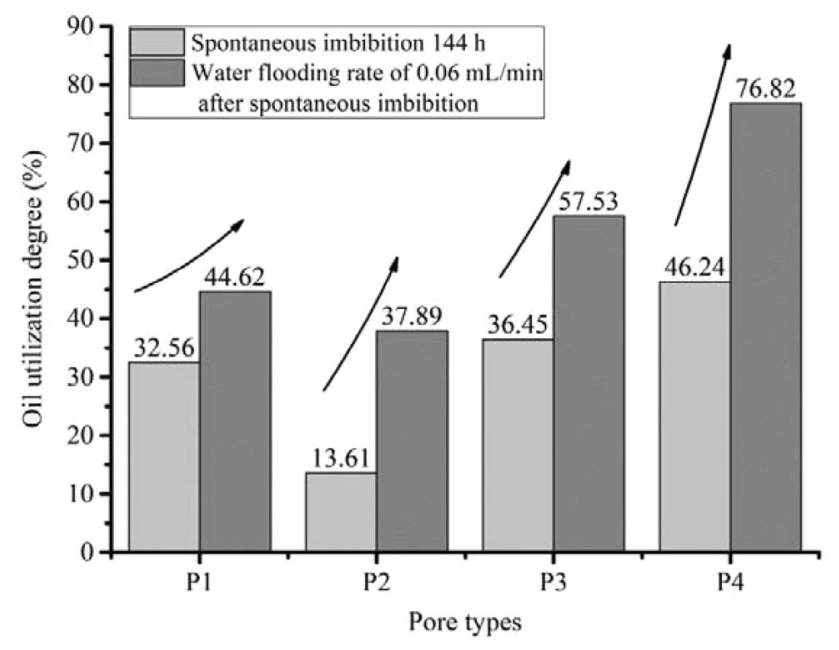

Fig. 13 OUD in various types of pores for the DBC81 sample at a water flooding rate of $0.06 \mathrm{~mL} / \mathrm{min}$ after SI

dominantly contributed during the initial period of the SI; with the continuous SI occurrences, the diffusion of the residual oil in the $\mathrm{P} 2, \mathrm{P} 3$, and $\mathrm{P} 4$ pores contributed to an increase in the oil displacement efficiency (Lyu et al. 2018b).

\section{OUD of various pores during the water flooding at various flooding rates}

A water flooding rate of $0.06 \mathrm{~mL} / \mathrm{min}$ could achieve the maximum oil displacement efficiency for the DBC81 and WQC91 samples, and this is related to the OUD of the pores. Evidently, the OUD of residual oil in the P2, P3, and $\mathrm{P} 4$ pores increased first and then decreased with the increase in the water flooding rates, and it reached maximum when the water flooding rate was $0.06 \mathrm{~mL} / \mathrm{min}$, while it fluctuated for the P1 pores (Fig. 12). The OUD for the P3 and P4 pores was significantly higher than that for the P2 pores owing to the larger pore aperture. However, the volume of residual oil in the $\mathrm{P} 2$ pores was dominant, and the characteristics of the OUD in P2 pores were essential.

\section{OUD of various pores in the water flooding displacement after SI}

It was found that a reasonable water flooding rate after SI could further increase the OUD of the residual oil in various types of pores, particularly for the $\mathrm{P} 2, \mathrm{P} 3 \mathrm{~s}$ and $\mathrm{P} 4$ pores in the DBC81 sample (Fig. 13). There was only a slight increase in the oil displacement efficiency for the WQC91 sample (Fig. 5b). The volume of various pores in the WQC91 sample is $\mathrm{P} 1: \mathrm{P} 2: \mathrm{P} 3: \mathrm{P} 4=1: 6.52: 0.41: 0.38$, whereas was $\mathrm{P} 1: \mathrm{P} 2: \mathrm{P} 3: \mathrm{P} 4=1: 3.74: 1.81: 1.39$ for the DBC81 sample, which leads to a lower OUD in the pores.

\section{OUD models under various displacement methods}

A tight reservoir features a complex pore structure, and various displacement methods lead to different oil displacement efficiencies. For the SI oil displacement, the residual oil in $\mathrm{P} 2$ and $\mathrm{P} 3$ pores dominantly contributed to the increase in the oil displacement efficiency; the OUD of residual oil in the $\mathrm{P} 2$ pores significantly influenced the oil displacement efficiency when the water flooding displacement method was used. For a reasonable water flooding rate displacement method after SI, the evident increase in the oil displacement efficiency primarily benefited from the massive use of residual oil in various pores, particularly in the P2, P3d and P4 pores, and the pore structure contributed to the OUD (Fig. 14).

\section{Conclusions}

1. The oil displacement efficiency of the tight sandstone for SI was low, and a reasonable water flooding rate produced the maximum oil displacement efficiency for the tight reservoir; in this study, the reasonable water flooding rate was approximately $0.06 \mathrm{~mL} / \mathrm{min}$. A reasonable water flooding rate after SI is an effective method for further improving the oil displacement efficiency.

2. Using the progressively refined pore classification method, the pores in the tight sandstone were divided into four types: P1, P2, P3, and P4 pores. For SI displacement, the residual oil in the $\mathrm{P} 2$ and $\mathrm{P} 3$ pores dominantly contributed to the increase in the oil displacement efficiency; the residual oil in the $\mathrm{P} 2$ pores had a significant influence on the increase in the oil displacement efficiency when water flooding was utilized; the use of a reasonable water flooding rate after SI could significantly increase the degree of utilization of residual oil in the various pores, particularly for the P2, P3, and P4 pore, and the pore structure also controlled the further increase in the oil displacement efficiency. 

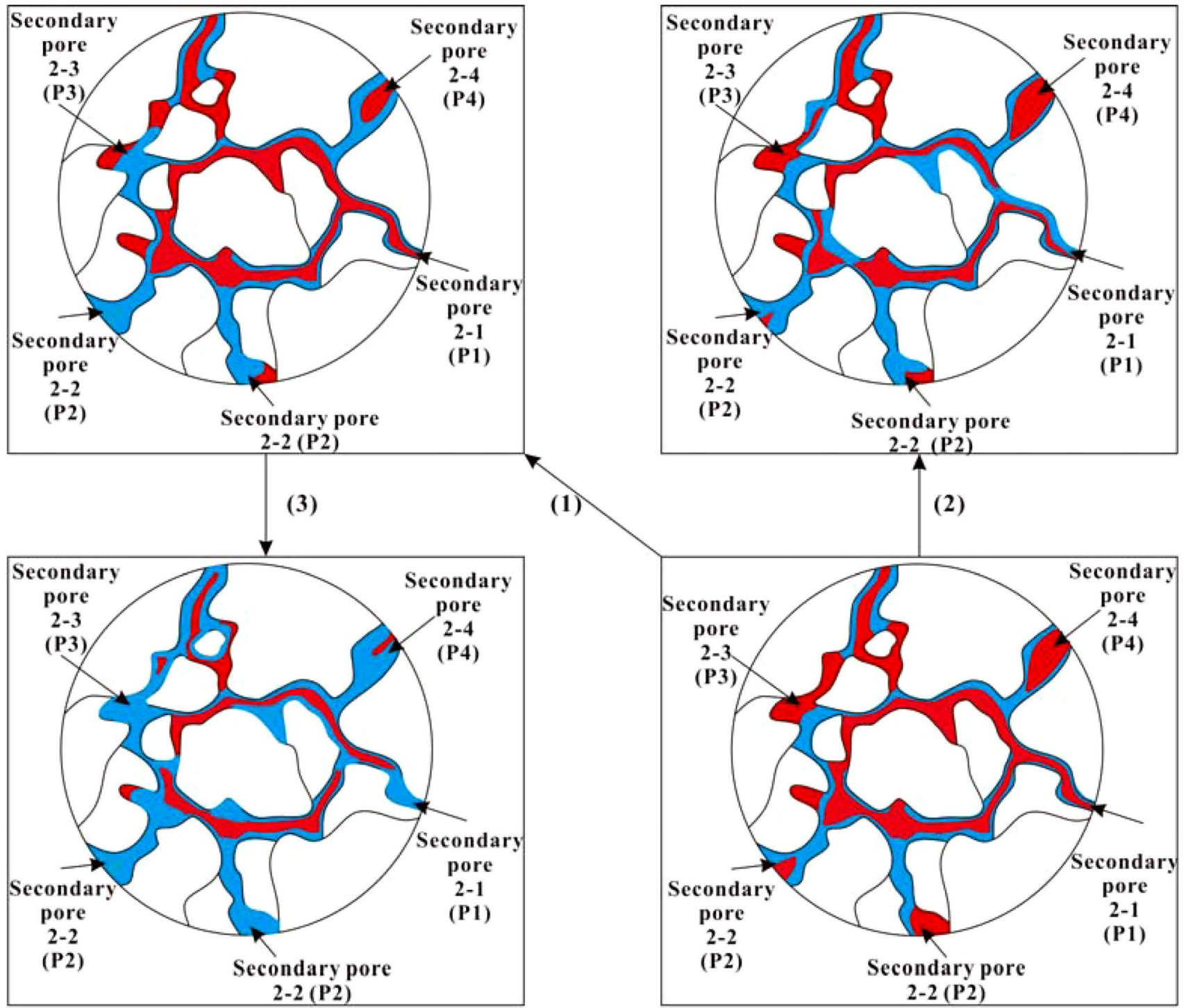

(1)

(2)

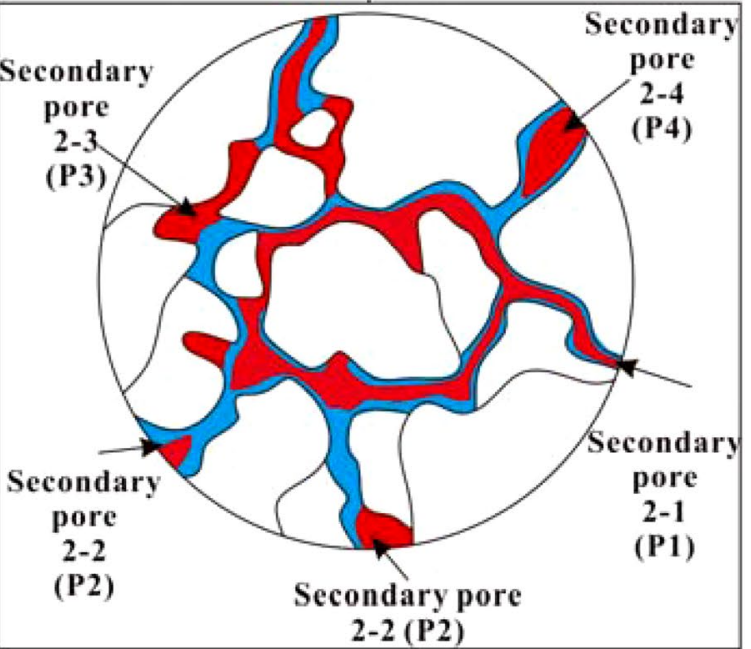

(1) Spontaneous imbibition oil displacement

(2) Water flooding under various rates

(3) Water flooding after spontaneous imbibition

Fig. 14 Diagram of the degree of utilization of residual oil using various displacement methods

Authors' contribution Corresponding author is Hui Gao, all the coauthors name order is Teng Li, Hui Gao, Chen Wang, Zhilin Cheng, Junjie Xue, Zhe Zhang, Kaiqing Luo, Ning Li, Xufei Liu, Jiangfeng Cao. Teng Li and Hui Gao contributed to Writing-original draft. Chen Wang and Zhilin Cheng contributed to measurements. Junjie Xue, Zhe Zhang, Kaiqing Luo, Ning Li, Xufei Liu and Jiangfeng Cao contributed to investigation.

Funding This work was supported by the National Natural Science Foundation of China (Program No. 52174030), Scientific Research Program Funded by Shaanxi Provincial Education Department (Program No. 21JP095 and Program No. 20JS116), Key Laboratory of Coalbed Methane Resources and Reservoir Formation Process of Ministry of
Education (China University of Mining and Technology) (Program No. 2021-004) and The Youth Innovation Team of Shaanxi Universities.

\section{Declarations}

Conflict of interest The authors declare that they have no known competing financial interests or personal relationships that could have appeared to influence the work reported in this paper.

Open Access This article is licensed under a Creative Commons Attribution 4.0 International License, which permits use, sharing, adaptation, distribution and reproduction in any medium or format, as long as you give appropriate credit to the original author(s) and the source, 
provide a link to the Creative Commons licence, and indicate if changes were made. The images or other third party material in this article are included in the article's Creative Commons licence, unless indicated otherwise in a credit line to the material. If material is not included in the article's Creative Commons licence and your intended use is not permitted by statutory regulation or exceeds the permitted use, you will need to obtain permission directly from the copyright holder. To view a copy of this licence, visit http://creativecommons.org/licenses/by/4.0/.

\section{References}

Al-Mahrooqi SH, Grattoni CA, Moss AK, Jing XD (2003) An investigation of the effect of wettability on NMR characteristics of sandstone rock and fluid systems. J Pet Sci Eng 39(3-4):389398. https://doi.org/10.1016/S0920-4105(03)00077-9

Chen M, Dai J, Liu X, Kuang Y, Wang Z, Gou S, Qin M, Li M (2020) Effect of displacement rates on fluid distributions and dynamics during water flooding in tight oil sandstone cores from nuclear magnetic resonance (NMR). J Pet Sci Eng 184:106588. https:// doi.org/10.1016/j.petrol.2019.106588

Cheng Z, Ning Z, Yu X, Wang Q, Zhang W (2019) New insights into spontaneous imbibition in tight oil sandstones with NMR. J Pet Sci Eng 179:455-464. https://doi.org/10.1016/j.petrol. 2019.04.084

Constantinides GN, Payatakes AC (1996) Network simulation of steady-state two-phase flow in consolidated porous media. AIChE J 42(2):369-382. https://doi.org/10.1002/aic.690420207

Cryer CW (1963) A comparison of the three-dimensional consolidation theories of Biot and Terzaghi. Quart J Mech Appl Math 16(4):401-412. https://doi.org/10.1093/qjmam/16.4.401

Dai C, Cheng R, Sun X, Liu Y, Zhou H, Wu Y, You Q, Zhang Y, Sun $Y$ (2019) Oil migration in nanometer to micrometer sized pores of tight oil sandstone during dynamic surfactant imbibitions with online NMR. Fuel 245:544-553. https://doi.org/10.1016/j.fuel. 2019.01.021

Gao Z, Hu Q (2016) Initial water saturation and imbibition fluid affect spontaneous imbibition into Barnett Shale samples. J Nat Gas Sci Eng 34:541-551. https://doi.org/10.1016/j.jngse.2016.07.038

Gao H, Wang C, Cao J, He M, Dou L (2019) Quantitative study on the stress sensitivity of pores in tight sandstone reservoirs of Ordos basin using NMR technique. J Pet Sci Eng 172:401-410. https:// doi.org/10.1016/j.petrol.2018.09.083

Garcia-Gutierrez C, Martinez FSJ, Caniego J (2017) A protocol for fractal studies on porosity of porous media: High quality soil porosity images. J Earth Sci 28:888-896. https://doi.org/10.1007/ s12583-017-0777-x

Ge HK, Liu Y, Shen YH, Ren K, Meng FB, Ji WM, Wu S (2015) Experimental investigation of shale imbibition capacity and the factors influencing loss of hydraulic fracturing fluids. Pet Sci 12:636-650. https://doi.org/10.1007/s12182-015-0049-2

Guo R, Kantzas A (2009) Assessing the water uptake of Alberta coal and the impact of $\mathrm{CO}_{2}$ injection with low-field NMR. J Can Pet Technol 48(7):40-46. https://doi.org/10.2118/09-07-40

Guo X, Huang Z, Zhao L, Han W, Ding C, Sun X, Yan R, Zhang T, Yang X, Wang R (2019) Pore structure and multi-fractal analysis of tight sandstone using MIP, NMR and NMRC methods: a case study from the Kuqa depression, China. J Pet Sci Eng 178:544 558. https://doi.org/10.1016/j.petrol.2019.03.069

Guo R, Xie Q, Qu X, Chu M, Li S, Ma D, Ma X (2020) Fractal characteristics of pore-throat structure and permeability estimation of tight sandstone reservoirs: a case study of Chang 7 of the Upper Triassic Yanchang Formation in Longdong area, Ordos Basin, China. J Pet Sci Eng 184:106555. https://doi.org/ 10.1016/j.petrol.2019.106555
Hamada G, AbuShanab MM (2008) Better porosity estimate of gas sandstone reservoirs using density and NMR logging data. Emirates J Eng Res 13(3):47-54. https://doi.org/10.2118/ 106627-MS

Hammecker C, Mertz JD, Fischer C, Jeannette D (1993) A geometrical model for numerical simulation of capillary imbibitions in sedimentary rocks. Trans Med 12:125-141. https://doi.org/10. 1007/BF00616976

Handy LL (1960) Determination of effective capillary pressures for porous media from imbibitions data. Petrol Trans AIME 219(1):75-80. https://doi.org/10.2118/1361-G

Hatiboglu CU, Babadagli T (2008) Pore-scale of spontaneous imbibiition into oil-saturated porous media. Phys Rev E 77(6):066311. https://doi.org/10.1103/PhysRevE.77.066311

Howard JJ, Kenyon WE, Straley C (1993) Proton-magnetic resonance and pore-size variations in reservoir sandstones. SPE Form Eval 8(3):194-200. https://doi.org/10.2118/20600-PA

Hu Y, Guo Y, Shangguan J, Zhang J, Song Y (2020) Fractal characteristics and model applicability for pores in tight gas sandstone reservoirs: a case study of the upper paleozoic in ordos basin. Energy Fuels 34(12):16059-16072. https://doi.org/10.1021/acs. energyfuels.0c03073

Jin Y, Dong J, Zhang X, Li X, Wu Y (2017a) Scale and size effects on fluid flow through self-affine rough fractures. Int J Heat Mass Transf 105:443-451. https://doi.org/10.1016/j.ijheatmasstrans fer.2016.10.010

Jin Y, Li X, Zhao M, Liu X, Li H (2017b) A mathematical model of fluid flow in tight porous media based on fractal assumptions. Int J Heat Mass Transfer 108(Part A):1078-1088. https://doi. org/10.1016/j.ijheatmasstransfer.2016.12.096

Kenyon WE, Day PI, Straley C, Willemsen JF (1988) A three part study of NMR longitudinal relaxation properties of water-saturated sandstones. SPE Form Eval 3(3):622-636. https://doi. org/10.2118/15643-PA

Khajepor S, Cui J, Dewar M, Chen B (2019) A study of wall boundary conditions in pseudopotential lattice Boltzmann models. Comput Fluids 193:103896. https://doi.org/10.1016/j.compf luid.2018.05.011

Kresse G, Hafner J (1993) Ab initio molecular dynamics for liquid metals. Phys Rev B 47(1):558-561. https://doi.org/10.1103/PhysR evB.47.558

Krohn CE (1988) Fractal measurements of sandstones, shales, and carbonates. J Geophys Res Solid Earth 93(B4):3297-3305. https:// doi.org/10.1029/JB093iB04p03297

Krohn CE, Thompson AH (1986) Fractal sandstone pores: automated measurements using scanning-electron-microscope images. Phys Rev B 33(9):6366-6374. https://doi.org/10.1103/PhysRevB.33. 6366

Lai J, Wang G, Wang Z, Chen J, Pang X, Wang S, Zhou Z, He Z, Qin Z, Fan X (2018) A review on pore structure characterization in tight sandstones. Earth Sci Rev 177:436-457. https://doi.org/10. 1016/j.earscirev.2017.12.003

Li K, Horne RN (2001) Characterization of spontaneous water imbibition into gas-saturated rocks. SPE J 6(4):375-384. https://doi.org/ 10.2118/74703-PA

Li P, Jia C, Jin Z, Liu Q, Zheng M, Huang Z (2019) The characteristics of movable fluid in the Triassic lacustrine tight oil reservoir: a case study of Chang 7 member of Xin'anbian Block, Ordos Basin, China. Mar Pet Geol 102:126-137. https://doi.org/10. 1016/j.marpetgeo.2018.11.019

Lyu C, Ning Z, Wang Q, Chen M (2018a) Application of NMR $T_{2}$ to pore size distribution and movable fluid distribution in tight sandstones. Energy Fuels 32(2):1395-1405. https://doi.org/10. 1021/acs.energyfuels.7b03431

Lyu C, Wang Q, Ning Z, Chen M, Li M, Chen Z, Xia Y (2018b) Investigation on the application of NMR to spontaneous imbibition 
recovery of tight sandstones: an experimental study. Energ 11(9):2359. https://doi.org/10.3390/en11092359

Mandelbrot BB (1979) Fractals: Form, chance, and dimension. J Am Stat 73(362):438. https://doi.org/10.2307/2286682

Mandelbrot BB, Passoja DE, Paullay AJ (1984) Fractal character of fracture surfaces of metal. Nature 308:721-722. https://doi.org/ $10.1038 / 308721 \mathrm{a} 0$

Martic G, Gentner F, Seveno D, Coulon D, De Coninck J (2002) A molecular dynamics simulation of capillary imbibitions. Langmuir 18(21):7971-7976. https://doi.org/10.1021/la020068n

Mattax CC, Kyte JR (1962) Imbibition oil recovery from fractured, water-drive reservoir. SPE J 2(2):177-184. https://doi.org/10. 2118/187-PA

Palma PRD, Huber C, Viotti P (2015) A new lattice Boltzmann model for interface reactions between immiscible fluids. Adv Water Resour 82:139-149. https://doi.org/10.1016/j.advwatres.2015. 05.001

Peng Y, Zhou C, Fan Y, Li C, Yuan C, Cong Y (2018) A new permeability calculation method using nuclear magnetic resonance logging based on pore sizes: a case study on bioclastic limestone reservoirs in the A oilfield of the Mid-Ease. Pet Explor Dev 45(1):183-192. https://doi.org/10.1016/S1876-3804(18)30019-3

Pfeifer P, Avnir D (1983) Chemistry in noninteger dimensions between two and three: I. Fractal theory of Heterogeneous surfaces. J Chem Phys 79(7):3558-3565. https://doi.org/10.1063/1.446210

Shao X, Pang X, Li H, Zhang X (2017) Fractal analysis of pore network in tight gas sandstones using NMR method: a case study from the Ordos Basin. China Energy Fuels 31(10):10358-10368. https:// doi.org/10.1021/acs.energyfuels.7b01007

Shen Y, Ge H, Li G, Yang X, Ren K, Yang Z, Su S (2016) Water imbibitions of shale and it potential influence on shale gas recovery-a comparative study of marine and continental shale formation. $\mathrm{J}$ Nat Gas Sci Eng 35:1121-1128. https://doi.org/10.1016/j.jngse. 2016.09.053

Tang L, Song Y, Jiang Z, Jiang S, Li Q (2019) Pore structure and fractal characteristics of distinct thermally mature shales. Energy Fuels 33(6):5116-5128. https://doi.org/10.1021/acs.energyfuels. $9 \mathrm{~b} 00885$

Volokitin Y, Looyestijn W, Slijkerman W, Hofman JP (2001) A practical approach to obtain primary drainage capillary pressure curves from NMR core and log data. Petrophysics 42(4):334-343

Wang X, Dang H, Gao T (2018) Method of moderate water injection and its application in ultra-low permeability oil reservoirs of Yanchang Oilfield. NW China Pet Explor Dev 45(6):1094-1102. https://doi.org/10.1016/S1876-3804(18)30112-5

Wang J, Cao Y, Liu K, Gao Y, Qin Z (2019a) Fractal characteristics of the pore structures of fine-grained, mixed sedimentary rocks from the Jimsar Sag, Junggar Basin: Implications for lacustrine tight oil accumulations. J Pet Sci Eng 182:106363. https://doi.org/10. 1016/j.petrol.2019.106363

Wang X, Hou J, Liu Y, Zhao P, Ma K, Wang D, Ren X, Yan L (2019b) Overall PSD and fractal characteristics of tight oil reservoirs: a case study of Lucaogou Formation in Junggar Basin. China
Fractals 27(1):1940005. https://doi.org/10.1142/S0218348X1 940005X

Wang J, Wu S, Li Q, Guo Q (2020) An investigation into pore structure fractal characteristics in tight oil reservoirs: a case study of the Triassic tight sandstone with ultra-low permeability in the Ordos Basin. China Arab J Geosic 13:961. https://doi.org/10.1007/ s12517-020-05928-0

Wang F, Zeng F, Wang L, Hou X, Cheng H, Gao J (2021) Fractal analysis of tight sandstone petrophysical properties in unconventional oil reservoirs with NMR and rate-controlled porosimetry. Energy Fuels 35(5):3753-3765. https://doi.org/10.1021/acs.energ yfuels.0c03394

Wu H, Zhang C, Ji Y, Liu R, Wu H, Zhang Y, Geng Z, Zhang Y, Yang J (2018) An improved method of characterizing the pore structure in tight oil reservoirs: Integrated NMR and constant-rate-controlled porosimetry data. J Pet Sci Eng 166:778-796. https://doi.org/10. 1016/j.petrol.2018.03.065

Wu B, Xie R, Wang X, Wang T, Yue W (2020) Characterization of pore structure of tight sandstone reservoirs based on fractal analysis of NMR echo data. J Nat Gas Sci Eng 81:103483. https://doi.org/10. 1016/j.jngse.2020.103483

Wu Y, Liu C, Ouyang S, Luo B, Zhao D, Sun W, Awan RS, Lu Z, Li G, Zang Q (2022) Investigation of pore-throat structure and fractal characteristics of tight sandstones using HPMI, CRMI, and NMR methods: A case study of the lower Shihezi Formation in the Sulige area, Ordos Basin. J Pet Sci Eng 210:110053. https:// doi.org/10.1016/j.petrol.2021.110053

Yang P, Guo H, Yang DT (2013) Determination of residual oil distribution during waterflooding in tight oil formations with NMR relaxometry measurements. Energy Fuels 27(10):5750-5756. https:// doi.org/10.1021/ef400631h

Yang L, Ge H, Shi X, Cheng Y, Zhang K, Chen H, Shen Y, Zhang J, $\mathrm{Qu} X$ (2016) The effect of microstructure and rock mineralogy on water imbibitions characteristics in tight reservoirs. J Nat Gas Sci Eng 34:1461-1471. https://doi.org/10.1016/j.jngse.2016.01.002

Yang Y, Wang D, Yang J, Wang B, Liu T (2021) Fractal analysis of CT images of tight sandstone with anisotropy and permeability prediction. J Pet Sci Eng 205:108919. https://doi.org/10.1016/j. petrol.2021.108919

Zeng F, Zhang Q, Guo J, Meng Y, Shao X, Zheng Y (2020) Capillary imbibitions of confined water in nanopores. Capillarity 3(1):8-15. https://doi.org/10.26804/capi.2020.01.02

Zhang J, Liu X, Wei Q, Sun K, Zhang G, Wang F (2017) Characterization of full-sized pore structure and fractal characteristics of marine-continental transitional Longtan Formation shale of Sichuan Basin. South China Energy Fuels 31(10):10490-10504. https://doi.org/10.1021/acs.energyfuels.7b01456

Publisher's Note Springer Nature remains neutral with regard to jurisdictional claims in published maps and institutional affiliations 